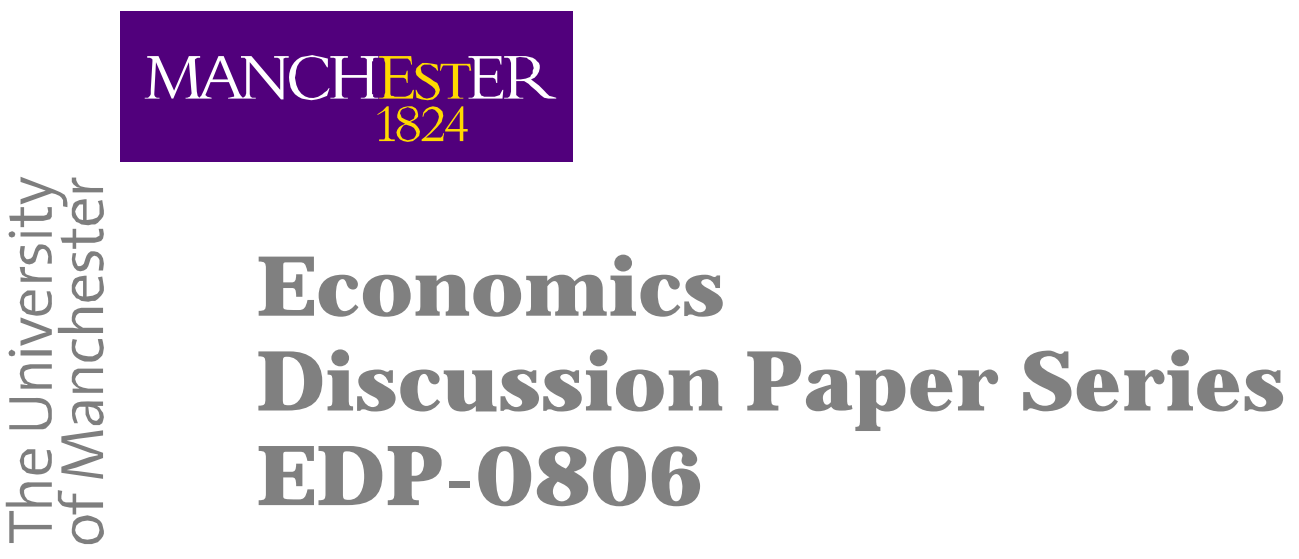

\title{
Risk aversion in symmetric and asymmetric contests
}

\author{
Richard Cornes \\ Roger Hartley
}

May 2008

Economics

School of Social Sciences

The University of Manchester

Manchester M13 9PL 


\title{
Risk aversion in symmetric and asymmetric contests
}

\author{
Richard Cornes \\ School of Economics \\ University of Nottingham \\ University Park \\ Nottingham \\ NG7 2RD \\ UK \\ rccornes@aol.com \\ Roger Hartley \\ Economic Studies \\ School of Social Sciences \\ University of Manchester \\ Oxford Road, Manchester \\ M13 9PL \\ UK \\ roger.hartley@manchester.ac.uk
}

May 8, 2008

\begin{abstract}
We analyze existence, uniqueness and properties of equilibria in incompletely discriminating Tullock contests with logistic contest success functions, when contestants are risk averse. We prove that a Nash equilibrium for such a contest exists, but give an example of a symmetric contest with both symmetric and asymmetric equilibria, showing that risk aversion may lead to multiple equilibria. Symmetric contests have unique symmetric equilibria but additional conditions are necessary for general uniqueness. We also study the effects on incumbents of additional competitors entering the contest under these
\end{abstract}


conditions and examine the effects of risk aversion on rent dissipation in symmetric and asymmetric contests.

Keywords: contest theory, aggregative noncooperative games, risk aversion

JEL classifications: C72, D72 


\section{Introduction}

Incompletely discriminating contests are widely used to analyze the consequences of rent-seeking behavior. In such a contest, contestants compete to win an indivisible rent with a common value to all contestants. Competition takes the form of choosing a level of expenditure and the profile of expenditure levels determines the probabilities of winning through a contest success function $^{1}$. The payoff of the winner is the value of the rent to that contestant net of the expenditure on rent seeking. The payoff of losers is the negative of their expenditure. This results in a simultaneous-move game in which strategies are expenditure levels, a player's payoff is her expected utility and we seek Nash equilibria. Such contests were introduced by Tullock [29] in part as a response to the competitive approach to rent seeking, which concluded that the whole value of the rent would be dissipated in rent-seeking activity. In these contests, dissipation is reduced both through strategic effects and as a consequence of the technology implicit in the contest success function. The study of such contests has blossomed into an extensive literature. Nitzan [19] and, more recently, Konrad [15] offer valuable surveys.

Most of the contest literature assumes that contestants are risk neutral. However, a number of authors have investigated the effects of risk aversion. Motivated partly by the dissipation research agenda, many of these papers compare equilibria under risk aversion with the corresponding contest in which players are risk neutral and investigate whether risk aversion reduces total expenditure on rent seeking. An early example is Hillman and Katz [11]. who work largely within the competitive paradigm characteristic of the early literature but also discuss some strategic issues in an appendix. They use a Taylor's series expansion to derive an expression for limiting rent dissipation in a symmetric contest when the rent is small, and adopt numerical methods to obtain some extensions of this to larger rents. Long and Vousden [16] discuss comparative statics and extensions to endogenous and divisible rents. Millner and Pratt [17], focussing on symmetric two-player contests, pointed out that risk-aversion need not reduce rent dissipation unless further restrictions are imposed on utility functions. Konrad and Schlesinger [14] show that this ambiguity extends to symmetric contests with any finite number of players and elucidate it by decomposing the risk effects of an increase in expenditure into a mean-preserving spread and contraction. These observations are in line with discussions of the effects of risk aversion on strategic effects in a wider class of games made by Skaperdas [24] and Grad-

\footnotetext{
${ }^{1}$ In an incompletely discriminating contest, this probability is less than one for the contestant with the greatest level of expenditure. The terminology is due to Hillman and Riley.
} 
stein [10]. Millner and Pratt also noted that, if the third derivative of the utility function is positive ${ }^{2}$, risk aversion will reduce equilibrium rent-seeking and carried out an experiment, the results of which were consistent with such a reduction. Their theoretical result was extended to more than two players in a recent note of Treich [28]. Existence of a Nash equilibrium was investigated by Skaperdas and Gan [26], who derived necessary and sufficient conditions for an equilibrium in two-player contests with constant absolute risk aversion and showed that, in some circumstances, risk aversion reduces expenditure on rent seeking. Cornes and Hartley [4] established existence and uniqueness of equilibrium in an asymmetric contest in which the coefficient of absolute risk aversion of contestants was constant. Bozhinov [3] extended this to constant relative risk aversion, but with a restriction on the size of the ratio of the size of the rent to initial wealth ${ }^{3}$. Cornes and Hartley also show that, given two otherwise identical contestants, the expenditure of the less risk averse contestant is greater and that global reductions in risk aversion increased aggregate lobbying. They also developed a formula for rent dissipation in a large symmetric contest and pointed out that selection effects in large contests may eliminate the more risk averse contestants, thereby partially offsetting the reduction in rent dissipation caused by risk aversion. Münster [18] studied the effects of risk aversion when contestants do not know how many other potential contestants have entered the contest. This leads to a game of incomplete information. Here, we confine the analysis to contests with complete information.

One focus of the general contest literature has been the existence and uniqueness of a pure-strategy equilibrium [27], [5]. With the exception of the special cases examined by Skaperdas and Gan [26], Cornes and Hartley [4] and Bozhinov [3], little attention has been paid to existence and uniqueness of equilibria in general contests with risk averse contestants. Indeed, most articles consider only symmetric equilibria of symmetric contests. Existence is complicated by inevitable discontinuities in payoffs at the origin (zero expenditure by all contestants.) Nevertheless, we show how other methods can be used to prove existence of an equilibrium for a wide class of contest success functions (those studied by Szidarowszky and Okuguchi [27]). If the contest is symmetric, it will have a unique symmetric equilibrium. However, we cannot rule out the possibility that such contests also have asymmetric

\footnotetext{
${ }^{2}$ This condition is now known as prudence [9], but Millner and Pratt's paper antedates this terminology.

${ }^{3}$ Since utility functions of contestants exhibiting constant relative risk aversion are only defined for positive arguments, initial wealth must exceed the rent. However, Bozhinov's upper bound on the ratio of rent to initial wealth is strictly less than one and depends on the coefficient of risk aversion.
} 
equilibria, nor of multiple equilibria of general contests. In particular, we present a symmetric example with a "proportional" contest success function (in which the probability that a contestant wins is equal to the ratio of the expenditure of that contestant to total expenditure) which possesses both symmetric and asymmetric equilibria. This shows that additional conditions (which we call "regularity") are needed to ensure uniqueness and a number of such conditions are exhibited in the sequel. All these conditions impose an upper bound on the curvature of the utility function and we show that this bound is always satisfied when the rent is small enough. In the case of constant relative risk aversion, we can give an explicit value for the rent, below which the contest admits a unique equilibrium. Cornes and Hartley [4] show that constant absolute risk aversion is sufficient for regularity and here we show that this remains true if the coefficient of absolute risk aversion is non-increasing provided this coefficient is not too large.

Comparative statics of contests have also been widely studied. For example, Long and Vousden [16] investigate contests in which contestants are risk averse and the rent is divisible. Nti [21] studies comparative statics for symmetric contests with risk-neutral contestants. For reasons of space, we restrict our analysis to the effects of entry, but permit the contestants to be risk-averse. In a symmetric contest, we show that, under the general conditions discussed above, entry reduces expenditure of incumbents in the symmetric equilibrium. In regular asymmetric contests, entry increases aggregate lobbying and decreases the probability that incumbents win as well as their payoffs in equilibrium.

We can use these results to analyze the effects of risk aversion on rent dissipation. Study of this issue was initiated by Tullock [29], who observed that strategic effects in a symmetric contest with $n$ contestants reduce the proportion of the rent dissipated from 1 under competition to (at most) ${ }^{4}$ $(n-1) / n$. A number of factors may further reduce rent dissipation, including asymmetry and the technology embodied in the contest success function $[5]^{5}$. For asymmetric contests with risk-averse contestants, we consider the case of contestants who are prudent and not too large (as measured by their equilibrium probability of winning.) Aggregate lobbying effort is smaller in such a contest than in a second contest with the same contest success function but risk-neutral contestants. This extends the recent result of Treich [28] for symmetric contests. When there are many contestants, the reduction

\footnotetext{
${ }^{4}$ The exact value depends on the contest success function and is equal to $(n-1) / n$ if this is proportional.

${ }^{5}$ Riley [22] discusses the role of asymmetry in reducing rent-seeking for completely discriminating winner-takes-all contests, where the whole rent is dissipated even for finitely many contestants.
} 
in dissipation caused by strategic effects vanishes $((n-1) / n$ approaches 1$)$ and studying the limit $n \longrightarrow \infty$ allows us to focus directly on the impact of risk aversion on rent dissipation. For symmetric contests, we derive an expression for the limiting dissipation ratio (proportion of the rent dissipated in equilibrium) in terms of the utility function. Special cases of this expression include the "small rent" formula of Hillman and Katz [11] as well as the limiting dissipation ratio found by Cornes and Hartley for the case of constant absolute risk aversion [4]. As in that paper, this limiting value is equal to one under risk neutrality and is strictly less than one under strict risk aversion. Furthermore, if two symmetric contests share the same contest success function but contestants in the second are more risk averse (in the Arrow-Pratt sense) than the first, then limiting rent dissipation is higher in the first contest.

Cornes and Hartley also studied the interaction between asymmetry in contests and risk aversion. Since asymmetry can also reduce rent dissipation, it might be expected that the reductions due to risk aversion and asymmetry will reinforce each other. However, the opposite will be true in large contests. With the proportional contest success function and many contestants exhibiting constant but different coefficients of risk aversion, competition will drive the more risk averse contestants out of the contest, leaving only the least risk averse contestants to participate actively in the contest. In the limit, rent dissipation approaches that associated with the smallest coefficient of risk aversion. With a more general contest success function, the story is a little more complicated: which contestants are inactive will depend on the contest success function as well as attitudes to risk. However, qualitative conclusions are essentially unchanged. Selection effects mean that, typically, the active contestants in a large contest exhibit the same attitudes to risk and success probability. This allows us to apply results derived for symmetric contests to large asymmetric contests.

To obtain these results we need a tractable characterization of Nash equilibria. The natural characterization is as a fixed point of the bestresponse mapping, but this mapping is multi-dimensional, which makes it hard to handle directly and for this reason, the existing literature on incompletely discriminating contests with risk aversion, except [4], assumes either symmetry or two contestants (or both). Instead, we use an alternative characterization in terms of share correspondences. This methodology was first discussed in [5], building on the share functions used in [4] to study contestants with constant absolute risk aversion and is ultimately derived from an approach to Cournot equilibrium pioneered by Selten [23]. The advantage of the approach is that much of the analysis is unidimensional. The method works because the game is aggregative (payoffs depend on own strategy and 
aggregate strategy) or, when contest success functions are not proportional, the contest is strategically equivalent to an aggregative game. This approach may have independent interest for other applications which can be modelled as aggregative games, including a number of extensions of the basic contest model.

The plan of the paper is as follows. In Section 2, we describe how the contest may be modelled as a simultaneous-move game and present an example which has a symmetric proportional contest success function and identical risk averse contestants yet possesses multiple equilibria. In the following section, we formally define share correspondences, derive some of their properties and establish existence of a Nash equilibrium. In Section 4, we first discuss uniqueness of symmetric equilibria of symmetric contests and then turn to additional conditions for ensuring a unique equilibrium of a general contest. In the following section, we study the effects of entry on equilibria, in both symmetric and asymmetric contests. In Section 6, after a brief look at small contests, we go on to develop an expression for the dissipation ratio for large symmetric contests and then extend this result to asymmetric contests. Section 7 concludes and proofs postponed from the main body of the text are given in the Appendix.

\section{Setup and counterexample}

We study a contest with $n(\geq 2)$ contestants of whom contestant $i$ chooses to spend $x_{i} \in \mathbb{R}_{+}$to influence the probability of winning an indivisible rent of value $R$. The expenditure $x_{i}$ purchases lobbying effort $f_{i}\left(x_{i}\right)$ where $f_{i}$ can be thought of as the production function of contestant $i$. We assume that all production functions are increasing and that production exhibits non-increasing returns.

A1 The production function $f_{i}$ is continuous, twice continuously differentiable in $\mathbb{R}_{+}$, and satisfies $f_{i}(0)=0, f_{i}^{\prime}(x)>0$ for $x \geq 0$ and $f_{i}^{\prime \prime}(x) \leq 0$ for $x>0$.

It is convenient to write $\mathbf{x} \in \mathbb{R}_{+}^{n}$ for the strategy profile $\left(x_{1}, \ldots, x_{n}\right)$ and we study contests with a single winner, in which the probability $p_{i}(\mathbf{x})$ that contestant $i$ wins is proportional to lobbying effort:

$$
p_{i}(\mathbf{x})=\frac{f_{i}\left(x_{i}\right)}{\sum_{j=1}^{n} f_{j}\left(x_{j}\right)} .
$$

This 'logistic' contest success function is widely used in the study of contests and was given an axiomatic foundation by Skaperdas [25]. If $f_{i}\left(x_{i}\right)=x_{i}$ 
for all $i$, we refer to the contest success function as proportional. In cases where $f_{i}$ is not defined for negative arguments, we interpret derivatives at the origin as one-sided and permit a limiting value of $+\infty$. This allows us to incorporate production functions such as $f_{i}(x)=x^{r}$ where $0<r \leq$ 1 , originally studied by Tullock [29] and in which the marginal product is unbounded.

We also suppose that contestants are risk averse or risk neutral with a concave Bernoulli utility function $u_{i}$ for contestant $i$, which satisfies the following conditions.

A2 Contestant $i$ has a utility function $u_{i}$, which is continuously differentiable and satisfies $u_{i}^{\prime}(c)>0$ and $u_{i}^{\prime \prime}(c) \leq 0$ for $c \in \mathbb{R}$.

Given a strategy profile $\mathbf{x} \neq \mathbf{0}$, contestant $i$ faces a gamble: win $R-x_{i}$ with probability $p_{i}$, lose $x_{i}$, otherwise. In this case, we take the payoff of contestant $i$ to be her expected utility:

$$
\tilde{\pi}_{i}(\mathbf{x})=\frac{f_{i}\left(x_{i}\right)}{\sum_{j=1}^{n} f_{j}\left(x_{j}\right)} u_{i}\left(R-x_{i}\right)+\left[1-\frac{f_{i}\left(x_{i}\right)}{\sum_{j=1}^{n} f_{j}\left(x_{j}\right)}\right] u_{i}\left(-x_{i}\right) .
$$

If the profile is $\mathbf{x}=\mathbf{0}$, we suppose that there is no winner and therefore take $\tilde{\pi}_{i}(\mathbf{0})=u_{i}(0)$ for all $i^{6}$. This defines an $n$-player simultaneous-move game in which player $i$ has strategy set $\mathbb{R}_{+}{ }^{7}$ and payoff $\tilde{\pi}_{i}$.

Note that $\widetilde{\pi}_{i}$ is discontinuous at the origin. Unfortunately, there is no way to define payoffs for all contestants that is continuous or even upper semicontinuous at the origin and that also respects the assumption that there is at most one winner ${ }^{8}$. This discontinuity at the origin implies that at least

\footnotetext{
${ }^{6} \mathrm{~A}$ natural alternative assumption is that every contestant wins with probability $1 / n$ when $\mathbf{x}=\mathbf{0}$. This makes no difference to our results.

${ }^{7}$ Since strategies exceeding $R$ are strictly dominated, we can take the strategy set to be $[0, R]$ without loss of generality. Indeed, there may be utility functions satisfying $\mathbf{A} 2$ only for $c \geq-R$, for which this may be the natural strategy set.

${ }^{8}$ The condition that there is at most one winner can be expressed as

$$
\sum_{i=1}^{n} \frac{\tilde{\pi}_{i}(\mathbf{x})-u_{i}\left(-x_{i}\right)}{u_{i}\left(R-x_{i}\right)-u_{i}\left(-x_{i}\right)} \leq 1
$$

Any attempt to define payoffs at the origin that are upper semi-continuous for all contestants and consistent with this inequality will encounter a contradiction. For, if $x_{j}=0$ for $j \neq i$, the payoff of contestant $i$ is $u_{i}\left(R-x_{i}\right)$ and this approaches $u_{i}(R)$ as $x_{i} \longrightarrow 0$. Upper semi-continuity of $\tilde{\pi}_{i}$ at $\mathbf{0}$ would dictate $\tilde{\pi}_{i}(\mathbf{0}) \geq u_{i}(R)$ for all $i$, but this would violate the displayed inequality at $\mathbf{x}=\mathbf{0}$ (and introduce $\mathbf{0}$ as a spurious equilibrium).
} 
two contestants must be active (choose positive $x_{i}$ ) in any Nash equilibrium. Indeed, if only contestant $i$ were active, we would have $\mathbf{x}_{-i}=\mathbf{0}$. But then, $\arg \max _{x_{i}} \tilde{\pi}_{i}\left(x_{i}, \mathbf{x}_{-i}\right)$ would be empty, contradicting the definition of equilibrium. The discontinuity also prevents us from direct use of standard existence theorems (e.g. the Debreu-Fan-Glicksberg pure-strategy existence theorem [7]). The fact that payoffs are not even upper semi-continuous prevents direct application of pure-strategy existence theorems such as those of Dasgupta and Maskin [6], which permit discontinuous payoffs. We return to this issue in the next section, where we prove that, nevertheless, A1 and A2 are sufficient to ensure existence of a Nash equilibrium.

When all players are risk neutral, this game is strategically equivalent to Cournot oligopoly with unit elastic demand and non-decreasing marginal costs. It is well known that such a game not only has a Nash equilibrium but that the equilibrium is unique [27], [5]. However, as the following counterexample shows, this result does not survive if risk neutrality is relaxed to risk aversion.

Example 1 Consider the 10-player contest, in which, for all $i$, we have $f_{i}(x)=x$ for $x \geq 0$ and $u_{i}$ satisfies $^{9}$

$$
u_{i}(c)=c-0.45 c^{2}
$$

for $0 \leq c \leq 1$. Assumption $\boldsymbol{A} \mathbf{1}$ obviously holds for all contestants and simple computation shows that $\boldsymbol{A} \mathscr{2}$ is satisfied with the displayed utility function. Direct calculation can be used to verify that this symmetric contest has a symmetric Nash equilibrium in which $x_{i}=0.0563$ for all $i . \quad$ (Numerical values throughout this example are given to three significant figures.) However, this equilibrium is not unique. For example, there are also asymmetric equilibria in which any three contestants choose $x_{i}=0.184$ and the remaining seven contestants choose $x_{i}=0$.

For analytical purposes it is helpful to rewrite the game using lobbying effort as strategic variable. Since $f_{i}$ is strictly increasing, $g_{i}=f_{i}^{-1}$ exists, is twice continuously differentiable in $\mathbb{R}_{+}$and satisfies $g_{i}(0)=0$ and $g_{i}^{\prime} \geq 0$ and $g_{i}^{\prime \prime} \geq 0$. Write $y_{i}=f_{i}\left(x_{i}\right)$ and $Y=\sum_{j=1}^{n} y_{j}$ for aggregate lobbying. Then we can rewrite the payoff of contestant $i$ as

$$
\pi_{i}\left(y_{i}, Y\right)=u_{i}\left[-g_{i}\left(y_{i}\right)\right]+\frac{y_{i}}{Y} D_{i}\left(y_{i}\right)
$$

\footnotetext{
${ }^{9}$ To make $\mathbb{R}_{+}$the strategy set, it is necessary to extend the definition of $u_{i}$ to $c \geq 1$ in such a way that $u_{i}$ is twice continuously differentiable, concave and increasing. This can easily be done without altering the set of equilibria.
} 
for $0 \leq y_{i} \leq Y$, where

$$
D_{i}\left(y_{i}\right)=\left\{u_{i}\left[R-g_{i}\left(y_{i}\right)\right]-u_{i}\left[-g_{i}\left(y_{i}\right)\right]\right\}>0,
$$

provided $Y>0$. Since $\mathbf{x}=\mathbf{0}$ cannot be an equilibrium, $Y$ is positive in all equilibria. Note that the transformed game is aggregative: each contestant's payoff depends only on their own strategy and the sum of all strategies, a fact we exploit throughout the development.

\section{Share correspondences}

Our analysis is rooted in the notion of a share correspondence. It extends the "fitting-in function" used by Selten [23], Bamón and Frayssé [1], Novshek [20] and others to study Cournot equilibrium and other aggregative games. The share correspondence of contestant $i$, denoted $\mathcal{S}_{i}$, is a mapping from $\mathbb{R}_{++}$to subsets of $[0,1]$. Fix the payoff of contestant $i$ to be $\pi_{i}\left(y_{i}, Y\right)$ and consider all aggregative games in which $i$ is a player. Consider further, all equilibrium strategy profiles of such a game in which aggregate lobbying effort is $Y$. Let $\mathcal{S}_{i}(Y)$ denote the set of probabilities that contestant $i$ wins in such an equilibrium:

$$
\mathcal{S}_{i}(Y)=\left\{\frac{y_{i}}{Y}: \mathbf{y} \text { is a Nash equilibrium with } \sum_{j=1}^{n} y_{j}=Y\right\} .
$$

Share correspondences can be used to study equilibria using the readilyproved fact that $\widehat{\mathbf{y}}$ is a Nash equilibrium if and only if $\widehat{y}_{i} / \widehat{Y} \in \mathcal{S}_{i}(\widehat{Y})$ for all $i$, where $\widehat{Y}=\sum_{j=1}^{n} \widehat{y}_{j}$. Equivalently, a necessary and sufficient condition (using standard set addition) for $\widehat{Y}$ to be an equilibrium value of aggregate lobbying in the contest is

$$
1 \in \sum_{j=1}^{n} \mathcal{S}_{j}(\widehat{Y})
$$

If this holds and $\sigma_{i} \in \mathcal{S}_{i}(\widehat{Y})$ for all $i$ satisfy $\sum_{j=1}^{n} \sigma_{j}=1$, then $\widehat{\mathbf{y}}=$ $\left(\sigma_{1} \widehat{Y}, \ldots, \sigma_{n} \widehat{Y}\right)$ is a Nash equilibrium.

Share correspondences can be characterized in terms of best-response mappings. Since the best response of contestant $i$ to the strategy profile $\mathbf{y}_{-i}$ depends only on $Y_{-i}=\sum_{j \neq i} y_{j}$, we can write the best-response correspondence as

$$
\mathcal{B}_{i}\left(Y_{-i}\right)=\arg \max _{y} \pi_{i}\left(y, y+Y_{-i}\right),
$$


for any $Y_{-i} \geq 0$. The share correspondence satisfies

$$
\sigma \in \mathcal{S}_{i}(Y) \Longleftrightarrow \sigma Y \in \mathcal{B}_{i}((1-\sigma) Y)
$$

We can use first-order conditions to rewrite this characterization since payoffs are quasi-concave functions of $y_{i}$ for fixed $Y_{-i} \geq 0$. (The proof is in the appendix.)

Lemma 3.1 If $\boldsymbol{A} 1$ and $\boldsymbol{A} \mathscr{2}$ hold for contestant $i$, then $\pi_{i}\left(y, y+Y_{-i}\right)$ is a quasi-concave function of $y \geq 0$ for any $Y_{-i} \geq 0$.

From (2), the marginal payoff can be written:

$$
\frac{\partial}{\partial y} \pi_{i}\left(y, y+Y_{-i}\right)=A_{i}\left(y_{i}, \frac{y_{i}}{y_{i}+Y_{-i}}\right)+\frac{Y_{-i}}{\left(y_{i}+Y_{-i}\right)^{2}} D_{i}\left(y_{i}\right),
$$

where

$$
A_{i}(y, \sigma)=-g_{i}^{\prime}(y)\left\{\sigma u_{i}^{\prime}\left[R-g_{i}(y)\right]+(1-\sigma) u_{i}^{\prime}\left[-g_{i}(y)\right]\right\} .
$$

It follows from Lemma 3.1 that $y_{i} \in \mathcal{B}_{i}\left(Y_{-i}\right)$ if and only if the right hand side of (6) is non-positive and equal to zero if $y_{i}>0$. Hence,

$$
\mathcal{S}_{i}(Y)=\left\{\sigma: 0 \leq \sigma<1 ; \phi_{i}(Y, \sigma) \leq 0 ; \sigma \phi_{i}(Y, \sigma)=0\right\},
$$

where $\phi_{i}$ is the marginal payoff expressed in terms of aggregate lobbying and share:

$$
\phi_{i}(Y, \sigma)=A_{i}(\sigma Y, \sigma)+\frac{1-\sigma}{Y} D_{i}(\sigma Y) .
$$

Note that $\phi_{i}(Y, 1)<0$ for any $Y>0$. If $\phi_{i}(Y, 0) \leq 0$, then $0 \in \mathcal{S}_{i}(Y)$. Alternatively, if $\phi_{i}(Y, 0)>0$, continuity implies that $\phi_{i}(Y, \sigma)=0$ for at least one $\sigma \in(0,1)$ and this puts $\sigma \in \mathcal{S}_{i}(Y)$. We conclude that share correspondences are non-empty for all $Y>0$. The following lemma, proved in the appendix, gives more information on these correspondences.

Lemma 3.2 Assume $\boldsymbol{A} 1$ and $\boldsymbol{A} \mathscr{2}$ hold for contestant $i$ and $\sigma \in \mathcal{S}_{i}(Y)$ for some $Y>0$. Then

1. $\sigma Y \leq f_{i}(R)$,

2. $0 \in \mathcal{S}_{i}(Y)$ if and only if

$$
Y \geq \bar{Y}_{i}=\frac{f_{i}^{\prime}(0)\left\{u_{i}(R)-u_{i}(0)\right\}}{u_{i}^{\prime}(0)},
$$


3. if (10) does not hold, $1-\sigma \leq K_{i} Y$, where

$$
K_{i}=\frac{u_{i}^{\prime}(-R)}{f_{i}^{\prime}(R)\left\{u_{i}(R)-u_{i}(0)\right\}} .
$$

We refer to $\bar{Y}_{i}$ as the dropout value of contestant $i$. Note that, if $f_{i}^{\prime}(0)=$ $\infty$, the dropout value is infinite and the second part of the lemma has the following corollary.

Corollary 3.1 Assume $\boldsymbol{A} \mathbf{1}$ and $\boldsymbol{A} \mathscr{2}$ hold for contestant $i$ and $f_{i}^{\prime}(0)=\infty$. Then $0 \notin \mathcal{S}_{i}(Y)$ for all $Y>0$.

Corollary 3.1 applies, for example, to all contestants in a Tullock contest in which $f_{i}(x)=x^{r}$ for all $i$, for some $0<r<1$. With this contest success function, every contestant will be active in equilibrium.

We can also use Lemma 3.2 to deduce properties of $\mathcal{S}_{i}(Y)$ for small and large $Y$ which will be useful in the sequel. In particular, the correspondence "approaches 1" as $Y \longrightarrow 0$ and "approaches 0 " as $Y \longrightarrow \infty$, in the sense of the following corollary, which follows directly from the first and third parts of the lemma.

Corollary 3.2 Assume A1 and A2 hold for contestant $i$. For any $\varepsilon>0$, there exist $Y^{-}$and $Y^{+}$such that $(i)$ if $\sigma \in \mathcal{S}_{i}(Y)$ and $0<Y<Y^{-}$, then $\sigma>1-\varepsilon$ and (ii) if $\sigma \in \mathcal{S}_{i}(Y)$ and $Y>Y^{+}$, then $\sigma<\varepsilon$.

It follows that all values in the image of the aggregate correspondence $\sum_{j=1}^{n} \mathcal{S}_{j}$ exceed 1 for small enough $Y$ and fall below 1 for all large enough $Y$. Existence turns on whether there is an intermediate value of $Y$ at which (5) holds and therefore an equilibrium exists. The proof of the following theorem may be found in the appendix.

Theorem 3.1 A contest in which $\boldsymbol{A} 1$ and $\boldsymbol{A} \mathscr{2}$ hold for all contestants has an equilibrium.

It is instructive to compare this theorem with the existence results of Skaperdas and Gan [26]. These authors consider contests with more general contest success functions than ours but with only two contestants, both exhibiting constant absolute risk aversion. With our contest success function, Skaperdas and Gan's sufficient conditions for existence are a special case of Theorem 3.1. In particular, constant absolute risk aversion can be relaxed to simple risk aversion. 


\section{Uniqueness}

Example 1 shows that $\mathbf{A} \mathbf{1}$ and $\mathbf{A} \mathbf{2}$ are insufficient on their own to exclude the possibility of multiple equilibria. In this section, we explore additional conditions for uniqueness.

\subsection{Symmetric contests}

We first consider symmetric equilibria of symmetric contests, noting that such equilibria are widely studied in the literature on contests. All contestants must be active in a symmetric equilibrium and win with probability $1 / n$, so a necessary and sufficient condition for $\widehat{Y}$ to be the value of aggregate lobbying in a symmetric equilibrium is that $1 / n \in \mathcal{S}(\widehat{Y})$, where $\mathcal{S}$ is the common share correspondence of all contestants. In the Appendix, we use the first order conditions to show that this occurs for exactly one value of $\widehat{Y}$.

Theorem 4.1 A symmetric contest in which $\boldsymbol{A} \mathbf{1}$ and $\boldsymbol{A} \mathscr{2}$ hold for all contestants has a unique symmetric equilibrium.

The applicability of this result is limited. Firstly, the contest must be symmetric. Even then, Example 1 shows that the contest may have multiple equilibria and a selection argument is needed to justify choosing the symmetric equilibrium, noting that every contestant may prefer some asymmetric equilibrium $^{10}$. For this reason, we now relax the restriction to symmetric equilibria.

\subsection{General contests}

Multiple equilibria can arise in two ways. Firstly, there can be several equilibria sharing a common value of $Y$. This is always the case where a symmetric contest has asymmetric equilibria as in Example 1. The second possibility, also illustrated by Example 1, is that different equilibria correspond to different values of $Y$. However, if $\mathcal{S}_{i}(Y)$ is a singleton for all positive $Y$, multiple equilibria of the first type are obviously ruled out. In such a case, the correspondence defines a share function $s_{i}$, where $\mathcal{S}_{i}(Y)=\left\{s_{i}(Y)\right\}$ for all $Y>0$ and (5) implies that $\widehat{Y}$ is an equilibrium value of aggregate

\footnotetext{
${ }^{10}$ It is straightforward to verify numerically that the payoff to an active player in the asymmetric equilibrium described in Example 1 is higher than that in the symmetric equilibrium.
} 
lobbying if and only if

$$
\sum_{j=1}^{n} s_{j}(\widehat{Y})=1 .
$$

This entails a unique equilibrium profile: $\left(s_{1}(\widehat{Y}) \widehat{Y}, \ldots, s_{n}(\widehat{Y}) \widehat{Y}\right)$. In a general aggregative game, multiple equilibria of the second type are still possible, but in contests with risk-averse (or risk-neutral) contestants, this cannot happen. This is a consequence of the following lemma, characterizing a number of useful properties of the share function and proved in the Appendix.

Lemma 4.1 Assume that $\boldsymbol{A} 1$ and $\boldsymbol{A} 2$ hold for contestant $i$ and $\mathcal{S}_{i}(Y)$ is a singleton $\left\{s_{i}(Y)\right\}$ for all $Y>0$. Then

1. $s_{i}$ is a continuous function;

2. $s_{i}$ is strictly decreasing where positive;

3. $s_{i}(Y) \longrightarrow 1$ as $Y \longrightarrow 0$.

4. If $f_{i}^{\prime}(0)$ is finite, $s_{i}(Y)=0$ if and only if $Y \geq \bar{Y}_{i}$. If $f_{i}^{\prime}(0)=\infty$, then $s_{i}(Y)>0$ for all $Y>0$ and $s_{i}(Y) \longrightarrow 0$ as $Y \longrightarrow \infty$.

When a share function $s_{i}$ exists for every contestant, it follows from the first and second parts of the lemma that the aggregate share function $\sum_{j=1}^{n} s_{j}$ is a continuous function that is strictly decreasing where positive. Furthermore, the third and fourth parts imply that it approaches $n$ as $Y \longrightarrow 0$ and approaches or is equal to zero as $Y \longrightarrow \infty$. We may conclude that (11) holds for exactly one value of $\widehat{Y}$ and therefore the contest has a unique Nash equilibrium.

Theorem 4.2 A contest in which $\boldsymbol{A} 1$ and $\boldsymbol{A} \mathscr{2}$ hold and $\mathcal{S}_{i}(Y)$ is a singleton for all $i$ and all $Y>0$ has a unique equilibrium.

We shall call contestant $i$ regular, if $\mathbf{A} \mathbf{1}$ and $\mathbf{A} \mathbf{2}$ are satisfied and, for all $Y>0$, there is a unique $\sigma \in[0,1]$ satisfying $\phi_{i}(Y, \sigma) \leq 0$ and $\sigma \phi_{i}(Y, \sigma)=0$, so $\mathcal{S}_{i}(Y)$ has one member. If all contestants are regular, we shall call the contest regular. Restating Theorem 4.2, a regular contest has a unique equilibrium. Example 1 shows that $\mathbf{A} \mathbf{1}$ and $\mathbf{A} \mathbf{2}$ alone are insufficient to ensure regularity. 
Example 2 (Example 1 revisited) In the contest discussed in Example 1 , we can calculate $\phi_{i}$ using $u_{i}(c)=c-0.45 c^{2}$, to find

$$
\phi_{i}(Y, \sigma)=-1+0.9 \sigma(1-Y)+\frac{1-\sigma}{Y}(0.55+0.9 \sigma Y) .
$$

It can be verified that $\phi_{i}(0.563,0)<0$ and $\phi_{i}(0.563,0.1)=0$ for all $i$ which means that no contestant is regular.

\subsection{Regularity}

In this subsection, we present sufficient conditions for regularity. Rather than impose additional restrictions on production functions, we focus on attitudes to risk and seek conditions on $u_{i}$ that ensure regularity for all production functions satisfying A1. In [4], Cornes and Hartley show that a contestant whose preferences are characterized by constant absolute risk aversion is regular. Bozhinov [3] extends this result to contests in which players exhibit constant relative risk aversion, but at the cost of imposing restrictions on initial wealth and the size of the rent. In Example 1, contestants are not regular, but exhibit increasing absolute risk aversion. These results suggest that non-increasing absolute risk aversion may be sufficient for regularity, but this conjecture remains to be settled. Note that prudence [9] (convex marginal utility), whilst implied by decreasing absolute risk aversion, is not itself sufficient for regularity. Indeed, the marginal utility in Example 1 is (weakly) convex. Furthermore, it is straightforward to perturb the utility function slightly in this example to construct a contest with multiple equilibria and strictly prudent contestants.

In the remainder of this section, we present a sufficient condition for regularity and then show that it is satisfied for constant relative risk aversion, provided the rent satisfies an upper bound and for decreasing absolute risk aversion provided that the coefficient of risk aversion is not too large.

Our principal sufficient condition imposes a restriction on the curvature of the utility function over the interval $(0, R)$. The proof may be found in the appendix.

Lemma 4.2 If $\boldsymbol{A} 1$ and $\boldsymbol{A} \mathscr{2}$ hold for contestant $i$ and

$$
d_{i}(x)=2 u_{i}^{\prime}(R-x)-u_{i}^{\prime}(-x) \geq 0
$$

for all $x \in(0, R)$, contestant $i$ is regular. 
To illustrate the application of this lemma, we consider the case of constant relative risk aversion:

$$
u_{i}(c)=\frac{\left(I_{i}+c\right)^{1-\mu_{i}}}{1-\mu_{i}} \text { where } \mu_{i}>0 \text { and } \mu_{i} \neq 1 .
$$

Here, $I_{i}$ is the initial wealth of contestant $i$. If $\mu_{i}=1$, we take $u_{i}=\ln \left(I_{i}+c\right)$. We assume $I_{i}>R$ and restrict strategies to $[0, R]$, ensuring that $I_{i}-x_{i}>0$ for all non-dominated strategies.

In this case,

$$
d_{i}(x)=2\left(I_{i}+R-x\right)^{-\mu_{i}}-\left(I_{i}-x\right)^{-\mu_{i}}
$$

and we look for the minimizer of $d_{i}$ in $[0, R]$. Firstly, we note that $d_{i}$ is a quasi-concave function of $x$ for $x \leq I_{i}$. This follows from the observation that, if

$$
d_{i}^{\prime}(x)=\mu_{i}\left[2\left(I_{i}+R-x\right)^{-\mu_{i}-1}-\left(I_{i}-x\right)^{-\mu_{i}-1}\right]=0,
$$

then

$$
\begin{aligned}
d_{i}^{\prime \prime}(x) & =\mu_{i}\left(\mu_{i}+1\right)\left[2\left(I_{i}+R-x\right)^{-\mu_{i}-2}-\left(I_{i}-x\right)^{-\mu_{i}-2}\right] \\
& =\mu_{i}\left(\mu_{i}+1\right)\left[2^{-1 /\left(\mu_{i}+1\right)}-1\right]\left(I_{i}-x\right)^{-\mu_{i}-2}<0 .
\end{aligned}
$$

We deduce that $d_{i}$ is minimized in $[0, R]$ at $x=0$ or $x=R$, so the condition in Lemma 4.2 holds if $d_{i}(0) \geq 0$ and $d_{i}(R) \geq 0$. The first of these inequalities can be re-arranged to

$$
R \leq\left(2^{1 / \mu_{i}}-1\right) I_{i}
$$

Similarly, $d_{i}(R) \geq 0$ gives

$$
R \leq\left(1-2^{-1 / \mu_{i}}\right) I_{i}<\left(2^{1 / \mu_{i}}-1\right) I_{i},
$$

where the second inequality can be justified by rearranging

$$
2^{-1 / \mu_{i}}\left(2^{1 / \mu_{i}}-1\right)^{2}>0 .
$$

The following corollary summarizes this conclusion.

Corollary 4.1 If $\boldsymbol{A} \boldsymbol{1}$ and $\boldsymbol{A} 2$ hold for contestant $i$ and $u_{i}$ is given by (12), where $I_{i} \geq R\left(1-2^{-1 / \mu_{i}}\right)^{-1}$, then contestant $i$ is regular.

This result shows that, if the size of the rent is not too large, the contest will have a unique Nash equilibrium. Such a result is true for more general utility functions, though we may not always be able to give an explicit formula 
for the bound on $R$. To support this claim, suppose that $u_{i}(c)=h_{i}\left(I_{i}+c\right)$, where $h_{i}$ is concave and continuously differentiable for positive arguments. Since $u_{i}^{\prime}$ is continuous,

$$
\min _{x \in\left[0, I_{i}\right]}\left\{2 u_{i}^{\prime}(R-x)-u_{i}^{\prime}(-x)\right\}
$$

is a continuous function of $R$. It is also positive for $R=0$ and therefore for all sufficiently small $R<I_{i}$. It follows that the condition in Lemma 4.2 holds for such $R$.

Corollary 4.2 Suppose that $\boldsymbol{A} \mathbf{1}$ and $\boldsymbol{A} \mathscr{2}$ hold for contestant $i$ and $u_{i}(z)=$ $h_{i}\left(I_{i}+z\right)$, where $I_{i}>0$ and $h_{i}$ is concave and continuously differentiable for positive arguments. Then there is $\underline{R} \in\left(0, I_{i}\right)$ such that contestant $i$ is regular for all $R<\underline{R}$.

The intuition behind this corollary is that risk-neutral contestants are regular and behavior for small gambles is approximately risk neutral. The corollary provides a formal demonstration of the smoothness implicit in this argument. Additionally, if the form of $h_{i}$ is known, it may be possible to use Lemma 4.2 to derive an explicit expression for $\underline{R}$.

We conclude this section with another corollary of Lemma 4.2.

Corollary 4.3 If $\boldsymbol{A} 1$ and $\boldsymbol{A} \mathscr{2}$ hold for prudent contestant $i$ and the coefficient of absolute risk aversion of this contestant does not exceed $1 / 2 R$ for any wealth level between $-R$ and $R$, contestant $i$ is regular.

Recall prudence means marginal utility is convex and therefore $u_{i}^{\prime \prime}\left(c_{1}\right) \leq$ $u_{i}^{\prime \prime}\left(c_{2}\right)$ whenever $c_{1} \leq c_{2}$. Hence, for any $x \in(0, R)$,

$$
2 u_{i}^{\prime}(R-x)-2 u_{i}^{\prime}(-x)=2 \int_{-x}^{R-x} u_{i}^{\prime \prime}(t) d t \geq 2 R u_{i}^{\prime \prime}(-x) \geq-u_{i}^{\prime}(-x),
$$

using the bound on the coefficient of risk aversion for the final inequality. Regularity follows from Lemma 4.2.

The fact that no upper bound is necessary when the coefficient of risk aversion is constant [4], suggest that the bound is not the best possible.

\section{$5 \quad$ Entry}

The approach used to study equilibrium existence and uniqueness can be adapted to analyze comparative statics, particularly with respect to the number of contestants. In this section, we discuss the effects on the equilibrium of adding and removing contestants. 


\subsection{Symmetric contests}

We first consider symmetric equilibria of symmetric contests. A general investigation of comparative statics for such equilibria has been conducted by Nti [21] for contests with risk neutral players. Nti considers changing the size of the rent as well as the number of contestants. For reasons of space, we concentrate on the latter here.

Let $Y^{n}$ denote the level of aggregate lobbying in the symmetric equilibrium, when the common production function $f$ and utility function $u$ satisfy assumptions $\mathbf{A} \mathbf{1}$ and $\mathbf{A 2}$, respectively. If a share function $s$ exists, $Y^{n}$ satisfies $n s\left(Y^{n}\right)=1$. Since $s$ is strictly decreasing where positive, $Y^{n}$ is increasing in $n$. If $f(x)=x$, then expenditure and lobbying are identical, so aggregate expenditure increases with $n$.

If no share function exists, $Y^{n}$ is uniquely determined by the consistency requirement $1 / n \in \mathcal{S}\left(Y^{n}\right)$. In contests where $\mathcal{S}$ need not be single-valued, this condition does not determine the ordering of $Y^{m}$ and $Y^{n}$ for $m<n$. If $Y^{m} \geq Y^{n}$, the lobbying effort of individuals falls as the size of the contest increases:

$$
y^{m}=\frac{Y^{m}}{m}>\frac{Y^{n}}{n}=y^{n},
$$

where $y^{m}$ is individual equilibrium lobbying. However, if $Y^{m}<Y^{n}$ both numerator and denominator are ordered in the same way, so we need a deeper analysis to compare $y^{m}$ and $y^{n}$. Nevertheless, a careful examination of the optimality conditions, conducted in the appendix, shows that we still have $y^{m}>y^{n}$ in the (unique) symmetric equilibrium. Since $f$ is strictly increasing by $\mathbf{A} \mathbf{1}$, we also have $x^{m}>x^{n}$.

Proposition 5.1 If $\boldsymbol{A} 1$ and $\boldsymbol{A} \mathscr{2}$ hold for all contestants in a symmetric contest, individual expenditures in the symmetric equilibrium decrease with the number of contestants. If, in addition, contestants are regular, aggregate lobbying increases and if, further, the contest success function is proportional, aggregate expenditure also increases.

\subsection{General contests}

In this subsection, we turn to asymmetric contests and assume throughout that all contestants are regular, so equilibria are unique. If an extra contestant enters such a contest, the aggregate share function increases. This increase is strict for values of $Y$ smaller than the entrant's dropout value. If $\widehat{Y}$, the pre-entry equilibrium value of $Y$, exceeds this dropout value, the entrant will be inactive and incumbents' equilibrium strategies will be unchanged. Otherwise, the new aggregate share function will exceed one at 
$Y=\widehat{Y}$. Since this function is strictly decreasing where positive, the new equilibrium value of $Y$ exceeds $\widehat{Y}$. Because individual share functions are non-increasing, the value of every share function decreases, strictly where positive. This implies that the probability that an active incumbent wins the contest falls and inactive incumbents remain inactive. In general, we cannot conclude from the increase in $Y$ that aggregate expenditure on rentseeking: $X=\sum_{j=1}^{n} x_{j}$ also increases because there is no direct relationship between $Y$ and $X$. Of course, if the contest success function is proportional, then $X=Y$, so $X$ increases even if contestants differ in their attitudes to risk.

No direct conclusion can be drawn about the effect of an increase in $Y$ on equilibrium strategies of individual contestants in asymmetric contests. This is because $y_{i}=Y s_{i}(Y)$ and $s_{i}$ decreases with $Y$ so $Y s_{i}(Y)$ need not be a monotonic function of $Y$. However, this does not prevent us from drawing conclusions about equilibrium payoffs as shown by the following lemma, proved in the appendix.

Lemma 5.1 Suppose $\boldsymbol{A} 1$ and $\boldsymbol{A} 2$ hold for contestant $i$ and this contestant is regular. If $\widetilde{Y}>Y>0$, then $\pi_{i}\left(Y s_{i}(Y), Y\right) \geq \pi_{i}\left(\widetilde{Y} s_{i}(\tilde{Y}), \tilde{Y}\right)$ and this inequality is strict if $s_{i}(Y)>0$.

It follows that an increase in $Y$ resulting from entry reduces the payoffs of active incumbents. The following result summarizes our discussion.

Proposition 5.2 Suppose that a regular contestant enters a regular contest and is active in the new equilibrium.

1. Aggregate lobbying strictly increases.

2. If the contest success function is proportional, aggregate expenditure strictly increases.

3. For any incumbent, the probability of winning and the payoff fall. This fall is strict for an incumbent that was active before entry.

\section{Dissipation}

The standard Tullock [29] rent-seeking contest was devised to study how strategy affects the proportion of the rent $R$ dissipated in attempts to win 
the rent. Specifically, for any equilibrium $\widehat{\mathbf{x}}$ of the contest, we define the dissipation ratio

$$
\rho=\frac{1}{R} \sum_{j=1}^{n} \widehat{x}_{j} .
$$

In a competitive free-entry model, the whole rent will be dissipated. In this section, we investigate the effect of risk aversion on $\rho$.

Konrad and Schlesinger [14] find that the dissipation ratio $\rho$ in a symmetric contest with risk-averse contestants need not be smaller than that in the corresponding contest in which contestants are risk-neutral. However, a recent note by Treich [28] shows that $\rho$ is indeed smaller if contestants in the former contest are also prudent ( $u_{i}^{\prime}$ is convex). Here, we extend Treich's result to asymmetric contests.

If $\mathcal{S}_{i}$ is the share correspondence of regular contestant $i$, we write $s_{i}^{\mathrm{N}}$ for the share function of a risk-neutral contestant with the same production function ${ }^{11}$. It follows from the first-order conditions defining $s_{i}$ that, in an obvious modification of the notation in (3) and $(7), D_{i}^{\mathrm{N}}(y)=R$ and $A_{i}^{\mathrm{N}}(y, \sigma)=-g_{i}^{\prime}(y)$. Hence, the marginal payoff of this contestant is

$$
\phi_{i}^{\mathrm{N}}(Y, \sigma)=-g_{i}^{\prime}(\sigma Y)+\frac{1-\sigma}{Y} R
$$

Comparing $\phi_{i}$ and $\phi_{i}^{\mathrm{N}}$ allows us to compare $\mathcal{S}_{i}$ and $s_{i}^{\mathrm{N}}$. The following lemma, proved in the appendix, gives the result.

Lemma 6.1 If $\boldsymbol{A} 1$ and $\boldsymbol{A} \mathscr{2}$ hold for prudent contestant $i$ and $\sigma \in \mathcal{S}_{i}(Y)$ satisfies $\sigma \leq 1 / 2$ for some $Y>0$, then $\sigma \leq s_{i}^{\mathrm{N}}(Y)$. If this contestant is strictly risk averse and $0<\sigma<1 / 2$, then $\sigma<s_{i}^{\mathrm{N}}(Y)$.

We can use the lemma to compare a contest with the corresponding contest in which all players are risk neutral. If $\widehat{\mathbf{y}}$ is an equilibrium profile of the former contest and $\widehat{Y}=\sum_{j} \widehat{y}_{j}$, then $\widehat{\sigma}_{i}=\widehat{y}_{i} / \widehat{Y} \in \mathcal{S}_{i}(\widehat{Y})$ for all $i$ and $\sum_{j} \widehat{\sigma}_{j}=1$. It follows from the lemma that, if all $\widehat{\sigma}_{i} \leq 1 / 2$, then $\sum_{j} s_{j}^{\mathrm{N}}(\widehat{Y}) \geq 1$. Consequently, $\widehat{Y}^{\mathrm{N}}$ the equilibrium lobbying aggregate of the latter contest, satisfies $\widehat{Y}^{\mathrm{N}} \geq \widehat{Y}$. Risk aversion reduces lobbying effort.

Proposition 6.1 Consider two contests with the same set of contestants and the same contest success function satisfying $\boldsymbol{A} 2$ for all contestants. Suppose all contestants satisfy $\boldsymbol{A} \mathbf{1}$ and are prudent in the first and risk neutral in the second.

\footnotetext{
${ }^{11} \mathrm{~A}$ risk-neutral contestant for which $\mathbf{A 1}$ holds is regular by Corollary 4.3 and therefore has a share function. A direct proof may be found in Cornes and Hartley[5].
} 
1. If no contestant wins the first contest with probability greater than $1 / 2$, equilibrium aggregate lobbying effort is greater in the second contest.

2. If, in addition, the contest success function is proportional, aggregate expenditure is greater in the latter contest.

3. If the contest is symmetric, individual and aggregate expenditures are greater in the latter contest [28].

The condition that $\widehat{\sigma}_{i} \leq 1 / 2$ for all $i$ holds in all equilibria of a contest in which there are at least two copies of each type of contestant and a fortiori in a symmetric contest. If contestants are strictly risk averse and winning probabilities are strictly less than $1 / 2$, the comparisons in the proposition are strict.

A fine-grained analysis of the effects of risk aversion is possible in large contests and, in the remainder of this section, we study such contests. For ease of exposition, we assume throughout the remainder of this section that $f_{i}^{\prime}(0)$ is finite ${ }^{12}$ for all $i$.

\subsection{Symmetric contests}

In a regular symmetric contest with $n$ contestants, it follows from Lemma 4.1 that the common share function, $s(Y)$, is continuous, decreases strictly to 0 at the common dropout value:

$$
\bar{Y}=\frac{u(R)-u(0)}{u^{\prime}(0)} f^{\prime}(0)
$$

and is equal to zero for $Y>\bar{Y}$. The aggregate share function $n s(Y)$ inherits these properties. Since $\widehat{Y}^{n}$, the equilibrium value of $Y$, satisfies $s\left(\widehat{Y}^{n}\right)=1 / n$, we conclude that $\widehat{Y}^{n} \longrightarrow \bar{Y}$ as $n \longrightarrow \infty$. The corresponding dissipation ratio satisfies

$$
\rho^{n}=\frac{\widehat{X}^{n}}{R}=\frac{n \widehat{x}^{n}}{R}=\frac{n g\left(\widehat{y}^{n}\right)}{R},
$$

where $\widehat{x}^{n}$ and $\widehat{X}^{n}$ are individual and aggregate expenditure and $\widehat{y}^{n}$ is individual lobbying (in equilibrium). Since $\widehat{y}^{n}=\widehat{Y}^{n} / n \longrightarrow 0$, we have

$$
\frac{g\left(\widehat{y}^{n}\right)-g(0)}{\widehat{y}^{n}} \longrightarrow g^{\prime}(0)
$$

\footnotetext{
${ }^{12}$ Note that, if $f_{i}^{\prime}(0)=\infty$, an arbitrarily small perturbation of $f_{i}$ will have finite slope at the origin. Specifically, $\widetilde{f}_{i}(x)=f_{i}(x+\varepsilon)$ satisfies $\widetilde{f}_{i}^{\prime}(0)<\infty$ for any $\varepsilon>0$.
} 
as $n \longrightarrow \infty$. Using $g(0)=0$ and $g^{\prime}(0)=\left[f^{\prime}(0)\right]^{-1}$, we deduce that $\rho^{n} \longrightarrow \widetilde{\rho}[u]$ as $n \longrightarrow \infty$, where

$$
\widetilde{\rho}[u]=\frac{u(R)-u(0)}{R u^{\prime}(0)} .
$$

A somewhat more involved argument, given in the appendix, shows that the latter conclusion is true even if contestants are not regular. Such contests may have multiple equilibria and therefore multiple values of the dissipation ratio. Nevertheless, if we select any one value for each $n$, the selected sequence tends to $\widetilde{\rho}[u]$.

Lemma 6.2 Let $\rho^{n}$ denote the dissipation ratio of an equilibrium of a symmetric contest in which the common production function $f$ satisfies $\boldsymbol{A} \mathbf{1}$ and $f^{\prime}(0)<\infty$ and the common utility function $u$ satisfies $\boldsymbol{A} \mathscr{2}$. Then $\rho^{n} \longrightarrow$ $\widetilde{\rho}[u]$ as $n \longrightarrow \infty$.

The limiting dissipation ratio $\widetilde{\rho}[u]$ is a measure of the curvature of the utility function. It can be viewed as the ratio of slope of the utility function between $R$ and 0 to the slope at the origin. Concavity of $u$ implies that $\widetilde{\rho}[u]$ increases to 1 (strictly if $u$ is strictly concave) as $R$ decreases to 0 . This is consistent with the conclusions of Hillman and Katz [11] for competitive rent-seeking ${ }^{13}$. Indeed, if we plug a third-order Taylor's series expansion for $u(R)$ into (16), we obtain

$$
\widetilde{\rho}[u]=1+\frac{R u^{\prime \prime}(0)}{2 u^{\prime}(0)}+\frac{R^{2} u^{\prime \prime \prime}(\widehat{R})}{6 u^{\prime}(0)},
$$

for some $\widehat{R} \in(0, R)$. This agrees (with appropriate change of notation) with the expression found by Hillman and Katz. These authors go on to conduct a numerical investigation of rent dissipation in both competitive and strategic models. It can be confirmed that $\widetilde{\rho}[u]$ agrees with the values that they find in both competitive and large strategic cases.

We have $\widetilde{\rho}[u]=1$ under risk neutrality and $\widetilde{\rho}[u]<1$ if $u$ is strictly concave. Indeed, more risk averse behavior (in the Arrow-Pratt sense) results in less of the rent being dissipated. Specifically, suppose that there is a differentiable, strictly increasing, strictly concave ${ }^{14}$ function $\psi$ such that $u^{*}(z)=\psi[u(z)]$ for all $z$. Strict concavity of $\psi$ implies

$$
\psi[u(R)]<\psi[u(0)]+\psi^{\prime}[u(0)][u(R)-u(0)] .
$$

\footnotetext{
${ }^{13}$ It also agrees with their strategic analysis, which assumes a proportional contest success function.

${ }^{14}$ This can be weakened to concave and nonlinear in $[0, R]$.
} 
It follows that

$$
\frac{u^{*}(R)-u^{*}(0)}{R u^{* \prime}(0)}=\frac{\psi[u(R)]-\psi[u(0)]}{R \psi^{\prime}[u(0)] u^{\prime}(0)}<\frac{u(R)-u(0)}{R u^{\prime}(0)},
$$

so $\widetilde{\rho}\left[u^{*}\right]<\widetilde{\rho}[u]$.

Corollary 6.1 For $n=2,3, \ldots$ let $\rho^{n}$ denote a dissipation ratio of a symmetric contest $\mathcal{C}^{n}$ satisfying the assumptions of Lemma 6.2. Let $\rho^{* n}$ denote a dissipation ratio of a symmetric contest with the same contest success function as $\mathcal{C}^{n}$ and in which the utility function satisfies $\boldsymbol{A} \mathscr{2}$ and represents more risk averse behavior than in $\mathcal{C}^{n}$. Then, $\rho^{* n}<\rho^{n}$ for all large enough $n$.

\subsection{General contests}

In a large asymmetric contest, Lemma 6.2 might suggest that dissipation would be an average of $\widetilde{\rho}[u]$ over all utility functions represented in the contest. However, there is an additional effect to consider. Increasing the size of the contest has a selection effect: contestants with smaller dropout values may be driven into inactivity. Hence, the limiting dissipation ratio need not be an average of $\widetilde{\rho}[u]$ over all utility functions in the contest. Rather, as the number of contestants increases, the selection effect biases the distribution towards contestants with larger dropout values.

We illustrate the point by considering a finite set of production functions: $f_{(1)}, \ldots, f_{(T)}$ satisfying $\mathbf{A} 1$ and $f_{(t)}^{\prime}(0)<\infty$ for all $t$ and utility functions: $u_{(1)}, \ldots, u_{(T)}$ satisfying A2. We study a sequence of contests: $\widetilde{\mathcal{C}}^{n}$ for $n=$ $2,3, \ldots$ in each of which $f_{i}=f_{(t)}, u_{i}=u_{(t)}$ (contestant $i$ is of type $t$ ) for some $t$ for each contestant $i$. We nest the contests by assuming that there are $m_{t}(n)$ contestants of type $t$ in $\widetilde{\mathcal{C}}^{n}$, where $m_{t}(n+1) \geq m_{t}(n)$ for $t=1, \ldots, T$ and $\sum_{t=1}^{T} m_{t}(n)=n$.

Without loss of generality, we can label the types so that $\bar{Y}_{(t)} \leq \bar{Y}_{(T)}$ for $t=1, \ldots, T-1$, where

$$
\bar{Y}_{(t)}=\frac{u_{(t)}(R)-u_{(t)}(0)}{u_{(t)}^{\prime}(0)} f_{(t)}^{\prime}(0) .
$$

Contestants of type $t$, satisfying $\bar{Y}_{(t)}<\bar{Y}_{(T)}$, are inactive in $\widetilde{\mathcal{C}}^{n}$ once there are enough contestants of type $T$. To see this when a share function exists for all types, note that the share function for type $T$ is strictly positive at $Y=\bar{Y}_{(t)}$ and, if $m_{(T)}(n)>\left[s_{(T)}\left(\bar{Y}_{(t)}\right)\right]^{-1}$, the aggregate share function exceeds one at $\bar{Y}_{(t)}$. Since share functions are strictly decreasing where positive, the equilibrium value of $Y$ exceeds $\bar{Y}_{(t)}$, so contestants of type $t$ 
are inactive. Even if share correspondences are multi-valued, we can modify this argument along the lines of the proof of Lemma 9.2 in the Appendix, to deduce that there is a positive constant $\delta>0$ such that $\sigma \geq \delta$ for all $\sigma \in \mathcal{S}_{(T)}\left(\bar{Y}_{(t)}\right)$. This implies that contestants of type $t$ are inactive if $m_{(T)}(n)>1 / \delta$. We conclude that, if $\bar{Y}_{(t)}<\bar{Y}_{(T)}$ for $t=1, \ldots, T-1$, only contestants of type $T$ are active once they are sufficiently numerous. We can therefore apply the results for symmetric contests to deduce that, the dissipation ratio approaches $\widetilde{\rho}\left[u_{(T)}\right]$ in this limit.

A complication with this argument arises if $\bar{Y}_{(t)}=\bar{Y}_{(T)}$ for some $t \neq T$, for then the dropout value $\bar{Y}_{(t)}$ depends on the marginal product $f_{(t)}^{\prime}(0)$ as well as the limiting dissipation ratio. As a result, if $\widetilde{\rho}\left[u_{(t)}\right] \neq \widetilde{\rho}\left[u_{(T)}\right]$, a mixture of types $t$ and $T$ will remain active in the limit and the mix of types will affect the limiting dissipation ratio. For simplicity of exposition, we ignore these complications by making the following assumption.

A3 Types are labelled so that for $t=1, \ldots, T-1$, either $\bar{Y}_{(t)}<\bar{Y}_{(T)}$, or both $\bar{Y}_{(t)}=\bar{Y}_{(T)}$ and $f_{(t)}^{\prime}(0)=f_{(T)}^{\prime}(0)$.

Type labels can always be chosen so that this assumption holds if all production functions are identical (symmetric contest success function) or if all utility functions are identical. Generically, A3 can always be satisfied: if $f_{(t)}^{\prime}(0)$ and $\widetilde{\rho}\left[u_{(t)}\right]$ are random draws from continuous distributions, the required labelling can be achieved with probability one.

Assuming $\mathbf{A 3}$ holds let $\mathcal{T}$ be the set of types satisfying $\bar{Y}_{(t)}=\bar{Y}_{(T)}$. If $m_{(T)}(n) \longrightarrow \infty$ as $n \longrightarrow \infty$, the equilibrium value of $Y$ approaches $\bar{Y}_{(T)}$. This allows us to determine the limiting dissipation ratio, for if $m_{(T)}(n)$ is sufficiently large that all types $t \notin \mathcal{T}$ are inactive, the dissipation ratio is

$$
\begin{aligned}
\frac{1}{R} \sum_{t \in \mathcal{T}} m_{(t)}(n) g_{(t)}\left(s_{(t)}\left(\widehat{Y}^{n}\right) \widehat{Y}^{n}\right) \\
\quad=\frac{\widehat{Y}^{n}}{R} \sum_{t \in \mathcal{T}} m_{(t)}(n) s_{(t)}\left(\widehat{Y}^{n}\right) \frac{g_{(t)}\left(s_{(t)}\left(\widehat{Y}^{n}\right) \widehat{Y}^{n}\right)}{s_{(t)}\left(\widehat{Y}^{n}\right) \widehat{Y}^{n}},
\end{aligned}
$$

where $\widehat{Y}^{n}$ is the equilibrium value of $Y$ in $\widetilde{\mathcal{C}}^{n}$. Since $\widehat{Y}^{n} \longrightarrow \bar{Y}_{(T)}=\bar{Y}_{(t)}$ for all $t \in \mathcal{T}$ we deduce that $s_{(t)}\left(\widehat{Y}^{n}\right) \widehat{Y}^{n} \longrightarrow 0$ as $n \longrightarrow \infty$. The equilibrium condition $\sum_{t \in \mathcal{T}} m_{(t)}(n) s_{(t)}(\widehat{Y})=1$ shows that the sum on the right hand side is a convex combination of terms each of which approaches $g_{(T)}^{\prime}(0)$ as $n \longrightarrow \infty$. (These limits are a consequence of $(15)$ for each type in $\mathcal{T}$.) We 
may deduce that the dissipation ratio approaches $\bar{Y}_{(T)} g_{(T)}^{\prime}(0) / R=\widetilde{\rho}\left[u_{(T)}\right]$ as $n \longrightarrow \infty$. Although we have assumed regularity in this argument, it is readily modified to allow for irregular contestants and share correspondences.

Proposition 6.2 Suppose $f_{(t)}$ satisfies $f_{(t)}^{\prime}(0)<\infty$ and $\boldsymbol{A 1}, u_{(t)}$ satisfies $\boldsymbol{A} 2$ for $t=1, \ldots, T, \boldsymbol{A} 3$ holds and $m_{(T)}(n) \longrightarrow \infty$. If $\rho^{n}$ is a dissipation ratio of the contest $\widetilde{\mathcal{C}}^{n}$, for $n=2,3, \ldots$, then $\rho^{n} \longrightarrow \widetilde{\rho}_{(T)}\left[u_{(T)}\right]$ as $n \longrightarrow \infty$.

If $f_{(t)}=f_{\left(t^{\prime}\right)}$ for all $t, t^{\prime}$, contest success functions of $\widetilde{\mathcal{C}}^{n}$ are symmetric and the ordering of dropout values is the same as that of limiting dissipation ratios $^{15}$. If one type of contestant is more risk averse than another, the former will have a smaller dissipation ratio and therefore smaller dropout value. Hence, they will be inactive for all large enough $n$. For example, if the risk attitudes of all contestants in a large contest with symmetric contest success function can be ordered in the Arrow-Pratt sense, dissipation ratios approach the dissipation ratio of the least risk averse contestant. This generalizes a result previously obtained for contestants with constant absolute risk aversion $[4]$.

\section{Conclusion}

We have offered a general treatment of common-value, incompletely discriminating contests in which contestants are risk averse. In particular, we show that, with risk averse contestants and production functions with nonincreasing returns, such contests have a Nash equilibrium. We also show that additional conditions are required to ensure that this equilibrium is unique and present several sufficient conditions for uniqueness. We study the effects of entry on aggregate lobbying and on incumbent contestants. Finally, we show that, in large contests, risk aversion reduces rent-seeking activity in both symmetric and asymmetric contests, though, in the latter, the effect may be offset by selection of active players in favor of less risk averse contestants. Similar conclusions may hold in smaller contests if we make additional assumptions: prudence and no contestant too large in equilibrium.

The techniques we have used above exploit the aggregative nature of the contest. A number of variations on the basic model also share this aggregative structure or are strategically equivalent to an aggregative game and can therefore potentially be addressed by the similar techniques. For example, Nti [21] introduces a "discount factor" in the form of an additive constant

\footnotetext{
${ }^{15}$ In fact, we only need $f_{(t)}^{\prime}(0)=f_{\left(t^{\prime}\right)}^{\prime}(0)$ for all $t, t^{\prime}$.
} 
in the denominator of the contest success function (1). Baye and Hoppe [2] observe that certain patent races are strategically equivalent to this form by interpreting hazard rates as production functions. If lobbying effort is used as the strategic variable, we still find that payoffs depend only on own strategy and aggregate strategy. Skaperdas and Gan [26] allow for limited liability which has the effect of making the payoff for losers independent of expenditure. This does not change the aggregative nature of the game. Konrad and Schlesinger [14] examine games in which the probability of winning is fixed but the size of the prize is a function of the expenditure profile. If this function takes a form similar to (1), the game is aggregative and may remain so when winning probabilities also depend on the expenditure profile, at least for some functional forms. The application of share correspondences and functions to such games awaits further investigation.

In Section 4, we presented a number of conditions ensuring uniqueness of equilibrium in contests with risk averse contestants and production functions satisfying A1. These appear to be some way from best possible. Cornes and Hartley [4] show that the equilibrium is unique if all contestants exhibit constant absolute risk aversion. The counterexample shows that this need no longer hold if the coefficient of risk aversion is increasing. This suggests the conjecture that contests in which all contestants exhibit non-increasing absolute risk aversion have a unique equilibrium. It follows from the analysis above that, if, for each $i$ and $Y>0$, the marginal payoff $\phi_{i}$ considered as a function of share $\sigma$ has at most one zero for $\sigma \in[0,1]$, contestant $i$ is regular. Since $\phi_{i}$ is negative at $\sigma=1$, this holds if $\phi_{i}$ is a quasiconvex function of $\sigma$. Numerical simulations for the case of constant relative risk aversion and covering a wide range of parameter values have all been consistent with quasi-convexity, even when the rent exceeds the bound in Corollary 4.1. However, we have not found a proof for this case, let alone the more general case of decreasing absolute risk aversion. To the best of our knowledge, the conjecture remains open.

\section{Bibliography}

\section{References}

[1] Bamón, R. and Frayssé, J. (1985), Existence of Cournot equilibrium in large markets, Econometrica, 53, pp 587-598.

[2] Baye, M. R. and Hoppe, H. C. (2003), The strategic equivalence of rent-seeking, innovation, and patent-race games, Games and Economic 
Behavior, 44, 2003, pp 217-226.

[3] Bozhinov, P. (2003), Rent-seeking by agents with constant relative risk aversion, mimeo, Keele University.

[4] Cornes, R. C. and Hartley, R. (2003), Risk aversion, heterogeneity and contests, Public Choice, 117, pp 1-25.

[5] Cornes, R. C. and Hartley, R. (2005), Asymmetric contests with general technologies, Economic Theory, 26, pp 923-946.

[6] Dasgupta, P. and Maskin, E. (1986), The existence of equilibrium in discontinuous economic games I: Theory, Review of Economic Studies, 53, pp 1-26.

[7] Fudenberg, D. and Tirole, J. (1991), Game Theory, MIT University Press, Cambridge, MA.

[8] Glicksberg, I. L. (1952), A further generalization of the Kakutani fixed point theorem with application to Nash equilibrium points, Proceedings of the National Academy of Sciences 38, pp 170-174.

[9] Eekhoudt, L. and Gollier, C. (2005), The impact of prudence on optimal prevention, Economic Theory, 26, pp989-994.

[10] Gradstein, M., Nitzan, S. and Slutzky, S. (1992), The effect of uncertainty on interactive behavior, Economic Journal, 102, pp 554-561.

[11] Hillman, A. L. and Katz, E. (1984), Risk-averse rent seekers and the social cost of monopoly power, Economic Journal, 94, pp 104-10.

[12] Hillman, A. L. and Samet, D. (1987), Dissipation of contestable rents by small numbers of contenders, Public Choice, 54, pp 63-82.

[13] Hillman, A. L. and Riley, J. G. (1989), Politically contestable rents and transfers, Economics and Politics, 1, pp 17-39.

[14] Konrad, K. A. and Schlesinger, H. (1997), Risk aversion in rent-seeking and rent-augmenting games, Economic Journal, 107, pp 1671-1683.

[15] Konrad, K. A. (2007), Strategy in contests - an introduction, WKZ Discussion Paper, SP II 2007-01.

[16] Long, N. and Vousden, N. (1987), Risk-averse rent seeking with shared rents, Economic Journal, 97, pp 971-985. 
[17] Millner, E. L. and Pratt, M. D. (1991), Risk aversion and rent-seeking: an extension and some experimental evidence, Public Choice, 69, pp $81-92$.

[18] Münster, J. (2006), Contests with an unknown number of contestants, Public Choice, 129, pp 353-368.

[19] Nitzan, S. (1994), Modelling rent-seeking contests, European Journal of Political Economy, 10, pp 41-60.

[20] Novshek, W. (1985), On the existence of Cournot equilibrium, Review of Economic Studies, 52, pp 85-98.

[21] Nti, K. (1997), Comparative statics of contests and rent-seeking games, International Economic Review, 38, pp 43-59.

[22] Riley, J. G. (2000), Asymmetric contests: a resolution of the Tullock paradox, in Howitt, P., De Antoni, E. and Leijonhufvud, A. (eds.) Money, Markets and Method: Essays in Honour of Robert W. Clower.

[23] Selten, R. (1970), Preispolitik der Mehrproduktunternehmung in der Statischen Theorie. Springer-Verlag, Berlin.

[24] Skaperdas, S. (1991), Conflict and attitudes towards risk, American Economic Review, 82, pp 720-739.

[25] Skaperdas, S. (1996), Contest success functions, Economic Theory, 7, pp 283-290.

[26] Skaperdas, S. and Gan, L. (1995), Risk aversion in contests, Economic Journal, 105, pp 951-962.

[27] Szidarowszky, F. and Okuguchi, K. (1997), On the existence and uniqueness of pure Nash equilibrium in rent-seeking games. Games and Economic Behavior, 18, pp 135-140.

[28] Treich, N. (2007), Risk-aversion (and prudence) in rent-seeking games, mimeo, LERNA-INRA, Toulouse School of Economics.

[29] Tullock, G. (1980), Efficient rent-seeking, in: Buchanan, J. M., Tollison, R. D. and Tullock, G. (eds.), Toward a Theory of the Rent-seeking Society, Texas A and M University Press pp131-46. 


\section{Appendix}

In this appendix, we give proofs postponed from above. The following expression for the derivative of $A_{i}$, defined in (7), is used in several of these proofs and is recorded here for convenience:

$$
\begin{aligned}
A_{i 1}(y, \sigma)= & \frac{\partial A_{i}}{\partial y}(y, \sigma) \\
= & \sigma D_{i}^{\prime \prime}(y)-g_{i}^{\prime \prime}(y) u_{i}^{\prime}\left[-g_{i}(y)\right]+\left[g_{i}^{\prime}(y)\right]^{2} u_{i}^{\prime \prime}\left[-g_{i}(y)\right] \\
= & -g_{i}^{\prime \prime}(y)\left\{\sigma u_{i}^{\prime}\left[R-g_{i}(y)\right]+(1-\sigma) u_{i}^{\prime}\left[-g_{i}(y)\right]\right\} \\
& +\left[g_{i}^{\prime}(y)\right]^{2}\left\{\sigma u_{i}^{\prime \prime}\left[R-g_{i}(y)\right]+(1-\sigma) u_{i}^{\prime \prime}\left[-g_{i}(y)\right]\right\} .
\end{aligned}
$$

Under assumptions $\mathbf{A 1}$ and $\mathbf{A 2}$, we deduce that

$$
A_{i 1}(y, \sigma) \leq 0
$$

Proof of Lemma 3.1. It follows from (6) that

$$
\frac{\partial}{\partial y} \pi_{i}\left(y, y+Y_{-i}\right)=-g_{i}^{\prime}(y) u_{i}^{\prime}\left[-g_{i}(y)\right]+\frac{Y_{-i}}{\left(y+Y_{-i}\right)^{2}} D_{i}(y)+\frac{y}{y+Y_{-i}} D_{i}^{\prime}(y),
$$

where $A_{i}(y, \sigma)$ and $D_{i}(y)$ are given in the expressions (7) and (3). Hence,

$$
\begin{aligned}
& \frac{\partial^{2}}{\partial y^{2}} \pi_{i}\left(y, y+Y_{-i}\right) \\
& \quad=-g^{\prime \prime}(y) u^{\prime}[-g(y)]+\left[g^{\prime}(y)\right]^{2} u^{\prime \prime}[-g(y)] \\
& -\frac{2 Y_{-i}}{\left(y+Y_{-i}\right)^{3}} D_{i}(y)+\frac{2 Y_{-i}}{\left(y+Y_{-i}\right)^{2}} D_{i}^{\prime}(y)+\frac{y}{y+Y_{-i}} D_{i}^{\prime \prime}(y) \\
& \quad=A_{i 1}\left(y, \frac{y}{y+Y_{-i}}\right)-\frac{2 Y_{-i}}{\left(y+Y_{-i}\right)^{3}} D_{i}(y)+\frac{2 Y_{-i}}{\left(y+Y_{-i}\right)^{2}} D_{i}^{\prime}(y),
\end{aligned}
$$

using (17) to obtain the second equality.

When $\partial \pi_{i} / \partial y=0$, the second derivative simplifies to

$$
\frac{\partial^{2}}{\partial y^{2}} \pi_{i}\left(y, y+Y_{-i}\right)=A_{i 1}\left(y, \frac{y}{y+Y_{-i}}\right)-\frac{2 g_{i}^{\prime}(y)}{y+Y_{-i}} u_{i}^{\prime}\left[R-g_{i}(y)\right]
$$

and we can use (18) to conclude that $\partial^{2} \pi_{i} / \partial y^{2} \leq 0$. So $\pi_{i}$ is quasi-concave. 
Proof of Lemma 3.2. It is straightforward to verify that any strategy $y_{i}$ satisfying $g_{i}\left(y_{i}\right)>R$ is strictly dominated by $y_{i}=0$. If follows that, if $y_{i}$ is a best response to any $Y_{-i} \geq 0$, then $y_{i} \leq g_{i}^{-1}(R)=f_{i}(R)$. The first part follows from the fact that $\sigma \in \mathcal{S}_{i}(Y)$ only if $\sigma Y$ is a best response.

The second part is a rearrangement of $\phi_{i}(Y, 0) \leq 0$, a necessary and sufficient condition for $0 \in \mathcal{S}_{i}(Y)$. It uses the fact that $f_{i}^{\prime}(0)=\left[g_{i}^{\prime}(0)\right]^{-1}$.

To prove the final part we start by observing that $D_{i}^{\prime}(y) \geq 0$, which implies

$$
D_{i}(\sigma Y) \geq D_{i}(0)=\frac{1-\sigma}{Y}\left\{u_{i}(R)-u_{i}(0)\right\} .
$$

From (18), $A_{i 1} \leq 0$, which gives the first inequality below

$$
\begin{aligned}
A_{i}(\sigma Y, \sigma) & \geq A_{i}\left(f_{i}(R), \sigma\right) \\
& =-g_{i}^{\prime}\left(f_{i}(R)\right)\left\{\sigma u_{i}^{\prime}\left[R-g_{i}\left(f_{i}(R)\right)\right]+(1-\sigma) u_{i}^{\prime}\left[-g_{i}\left(f_{i}(R)\right)\right]\right\} \\
& =-\left\{\sigma u_{i}^{\prime}(0)+(1-\sigma) u_{i}^{\prime}(-R)\right\} / f_{i}^{\prime}(R) \\
& \geq-u_{i}^{\prime}(-R) / f_{i}^{\prime}(R)
\end{aligned}
$$

in the remaining lines we have used (7) for the first equality, $g_{i}\left(f_{i}(R)\right)=R$ and $g_{i}^{\prime}\left(f_{i}(R)\right)=1 / f_{i}^{\prime}(R)$ for the second equality as well as $u_{i}^{\prime}(0) \leq u_{i}^{\prime}(-R)$ (a consequence of $\mathbf{A 2}$ ) for the third inequality. If (10) does not hold, $\sigma>0$ and so $\phi_{i}(Y, \sigma)=0$. Hence, using (3) and the concavity of $u$, we have

$$
-\frac{u_{i}^{\prime}(-R)}{f_{i}^{\prime}(R)}+\frac{1-\sigma}{Y}\left\{u_{i}(R)-u_{i}(0)\right\} \leq A_{i}(\sigma Y, \sigma)+\frac{1-\sigma}{Y} D_{i}(\sigma Y)=0 .
$$

This can be rearranged to give the inequality in the Lemma.

Proof of Theorem 3.1. Consider a sequence of numbers $\varepsilon^{m} \in\left(0, f_{i}(R)\right)$ satisfying $\varepsilon^{m} \longrightarrow 0$ as $m \longrightarrow \infty$. We start by modifying the contest by restricting the strategies of contestant $i$ to satisfy $\varepsilon \leq y_{i} \leq f_{i}(R)$. Since payoffs are continuous in all strategies and quasi-concave by Lemma 3.1, we can apply a standard existence theorem [7] to deduce that there exists an equilibrium strategy profile $\widehat{\mathbf{y}}^{m}$ for each $m$ and write $\widehat{Y}^{m}$ for $\sum_{j} \widehat{y}_{j}^{m}$. Since the corresponding sequence of share profiles $\left(\sigma_{1}^{m}, \ldots, \sigma_{n}^{m}\right)$ lies in the (compact) $n$-simplex, we can assume without loss of generality that there is some $\left(\sigma_{1}^{*}, \ldots, \sigma_{n}^{*}\right)$ in the simplex, such that $\sigma_{i}^{m} \longrightarrow \sigma_{i}^{0}$ as $m \longrightarrow \infty$. The first-order conditions for the $m$ th contest imply

$$
\phi_{i}\left(\widehat{Y}^{m}, \sigma_{i}^{m}\right) \leq 0 \text { and }\left(\widehat{Y}^{m} \sigma_{i}^{m}-\varepsilon^{m}\right) \phi_{i}\left(\widehat{Y}^{m}, \sigma_{i}^{m}\right)=0 \text { for } i=1, \ldots, n \text {. }
$$


Since $0 \leq \widehat{Y}^{m} \leq \sum_{j=1}^{m} f_{j}(R)$, we can deduce, by restricting to a subsequence if necessary, the existence of $\widehat{Y}^{*} \geq 0$ such that $\widehat{Y}^{m} \longrightarrow \widehat{Y}^{*}$ as $m \longrightarrow \infty$. If $\widehat{Y}^{*}>0$, we can take the limit $m \longrightarrow \infty$ and use the continuity of $\phi_{i}$ to deduce that the first-order conditions for $\sigma_{i}^{*} \in \mathcal{S}_{i}\left(\widehat{Y}^{*}\right)$ hold for $i=1, \ldots, m$. Since we also have $\sum_{j=1}^{m} \sigma_{j}^{*}=1$, the equilibrium condition is satisfied and existence of an equilibrium is established. The proof is completed by showing that we cannot have $\widehat{Y}^{*}=0$.

We shall show that $\widehat{Y}^{m} \longrightarrow 0$ leads to a contradiction. To see this, note first that $\sum_{j=1}^{n} \sigma_{j}^{*}=1$ implies that $\sigma_{\hat{\imath}}^{*}<1$ for some contestant $\hat{\imath}$ and therefore $\sigma_{\hat{\imath}}^{m}<1$ for all large enough $m$. Then,

$$
\phi_{\hat{\imath}}\left(\widehat{Y}^{m}, \sigma_{\hat{\imath}}^{m}\right)=A_{\hat{\imath}}\left(\sigma_{\hat{\imath}}^{m} \widehat{Y}^{m}, \sigma_{\hat{\imath}}^{m}\right)+\frac{1-\sigma_{\hat{\imath}}^{m}}{\widehat{Y}^{m}} D_{\hat{\imath}}\left(\sigma_{\hat{\imath}}^{m} \widehat{Y}^{m}\right) .
$$

Since $A_{\hat{\imath}}\left(\sigma_{\hat{\imath}}^{m} \widehat{Y}^{m}, \sigma_{\hat{\imath}}^{m}\right)$ has a finite limit and $D_{\hat{\imath}}\left(\sigma_{\hat{\imath}}^{m} \widehat{Y}^{m}\right) \longrightarrow D_{\hat{\imath}}(0)>0$ as $m \longrightarrow \infty$, we deduce that $\phi_{\hat{\imath}}\left(\widehat{Y}^{m}, \sigma_{\hat{\imath}}^{m}\right)>0$ for all large enough $m$. This contradicts (19).

The proof of Theorem 4.1 uses the following lemma, which is also used to establish Lemma 4.1.

Lemma 9.1 If $\boldsymbol{A} \boldsymbol{1}$ and $\boldsymbol{A} \mathscr{2}$ hold for contestant $i$ and $\phi_{i}(Y, \sigma)=0$, then $\partial \phi_{i}(Y, \sigma) / \partial Y<0$.

Proof. Using $D_{i}$ as defined in (3) and $A_{i 1}$ in (17), we have

$$
\frac{\partial \phi_{i}(Y, \sigma)}{\partial Y}=\sigma A_{i 1}(\sigma Y, \sigma)+\sigma \frac{1-\sigma}{Y} D_{i}^{\prime}(\sigma Y)-\frac{1-\sigma}{Y^{2}} D_{i}(\sigma Y) .
$$

If $\phi_{i}(Y, \sigma)=0$, then

$$
\frac{\partial \phi_{i}(Y, \sigma)}{\partial Y}=\sigma A_{i 1}(\sigma Y, \sigma)+\sigma \frac{1-\sigma}{Y} D_{i}^{\prime}(\sigma Y)+\frac{A_{i}(\sigma Y, \sigma)}{Y} .
$$

Using (18): $A_{i 1} \leq 0$, we find, after some rearrangement,

$$
\frac{\partial \phi_{i}(Y, \sigma)}{\partial Y} \leq-\frac{g_{i}^{\prime}(\sigma Y)}{Y}\left\{\sigma(2-\sigma) u_{i}^{\prime}\left[R-g_{i}(\sigma Y)\right]+(1-\sigma)^{2} u_{i}^{\prime}\left[-g_{i}(\sigma Y)\right]\right\} .
$$

For $0<\sigma \leq 1$, we have $\sigma(2-\sigma)>0$, so $\partial \phi_{i}(Y, \sigma) / \partial Y<0$.

Proof of Theorem 4.1. Since all contestants are active in a symmetric equilibrium, any equilibrium value of $Y$ must satisfy $\phi_{1}(\widehat{Y}, 1 / n)=0$. Since, 
$\phi_{1}(Y, 1 / n)$ is a continuous function, it follows immediately from Lemma 9.1 that $\phi_{1}(\widehat{Y}, 1 / n)=0$ has at most one solution. By Theorem 3.1, there is exactly one solution and there is a unique equilibrium in which $y_{i}=\widehat{Y} / n$ for all $i$.

Proof of Lemma 4.1. Continuity of $s_{i}$ for positive $Y$ follows by a standard compactness argument utilizing the fact that $\sigma=s_{i}(Y)$ if and only if $(Y, \sigma)$ satisfies

$$
\phi_{i}(Y, \sigma) \leq 0 \text { and } \sigma \phi_{i}(Y, \sigma)=0 .
$$

(Suppose $Y^{n} \longrightarrow Y^{0}$ as $n \longrightarrow \infty$ and consider a subsequence of the sequence $\left\{s_{i}\left(Y^{n}\right)\right\}$ convergent to some $\sigma^{0} \in[0,1]$. Using the facts that $\left(Y^{n}, s_{i}\left(Y^{n}\right)\right)$ satisfies (20) for all $n$ and that $\phi_{i}(Y, \sigma)$ is continuous for $0 \leq \sigma \leq 1$ and $Y>0$, we can take limits in $(20)$ on the subsequence to deduce that $\left(Y^{0}, \sigma^{0}\right)$ satisfies (20). Hence, $\sigma^{0}=s_{i}\left(Y^{0}\right)$. Since $s_{i}\left(Y^{n}\right)$ lies in the compact set $[0,1]$ for all $n$, we may conclude that $s_{i}\left(Y^{n}\right) \longrightarrow s_{i}\left(Y^{0}\right)$ as $n \longrightarrow \infty$.

The limits of $s_{i}$ as $Y \longrightarrow 0$ and $Y \longrightarrow \infty$ are immediate consequences of Corollary 3.2.

That the share function is zero if and only if $Y \geq \bar{Y}_{i}$ holds is a simple restatement of the second part of Lemma 3.2 for a singleton-valued share correspondence. The assertion for the case $f_{i}^{\prime}(0)=\infty$ is a direct consequence of the first and second parts of the lemma.

It remains to establish that $s_{i}$ is strictly decreasing where positive and, since $s_{i}$ is a continuous function, it is enough to show that, if $s_{i}(Y)>0$, there is a $Y^{\prime}>Y$ such that $s_{i}\left(Y^{\prime \prime}\right)<s_{i}(Y)$ for all $Y^{\prime \prime} \in\left(Y, Y^{\prime}\right)$. To do this, note that $\phi_{i}\left(Y, s_{i}(Y)\right)=0$ and therefore Lemma 9.1 implies there is a $Y^{\prime}>Y$ such that, if $Y<Y^{\prime \prime}<Y^{\prime}$, then $\phi_{i}\left(Y^{\prime \prime}, s_{i}(Y)\right)<0$. There are now two possibilities. One possibility is that $\phi_{i}\left(Y^{\prime \prime}, 0\right) \leq 0$, in which case $s_{i}\left(Y^{\prime \prime}\right)=0<s_{i}(Y)$. Alternatively, we must have $\phi_{i}\left(Y^{\prime \prime}, 0\right)>0$, in which case, since $\phi_{i}\left(Y^{\prime \prime}, s_{i}(Y)\right)<0$ and $\phi_{i}\left(Y^{\prime \prime}, \sigma\right)=0$ for a unique $\sigma=s_{i}\left(Y^{\prime \prime}\right)$, we must have $s_{i}\left(Y^{\prime \prime}\right)<s_{i}(Y)$. This completes the proof.

Proof of Lemma 4.2. It is clear that contestant $i$ will be regular if $\phi_{i}(Y, \sigma)$ is a strictly decreasing function of $\sigma \in(0,1)$ where it crosses the axis: $\phi_{i}(Y, \sigma)=0$. To prove that this is the case, use (7) to eliminate $A_{i}$ from the expression for $\phi_{i}$ and differentiate with respect to $\sigma$, holding $Y$ fixed 
to get,

$$
\begin{aligned}
\frac{\partial \phi_{i}}{\partial \sigma}= & -Y g_{i}^{\prime \prime}(\sigma Y) u_{i}^{\prime}\left[-g_{i}(\sigma Y)\right]+Y\left[g_{i}^{\prime}(\sigma Y)\right]^{2} u_{i}^{\prime \prime}\left[-g_{i}(\sigma Y)\right] \\
& +\sigma Y D_{i}^{\prime \prime}(\sigma Y)-\frac{1}{Y} D_{i}(\sigma Y)+(2-\sigma) D_{i}^{\prime}(\sigma Y), \\
= & Y A_{i 1}(\sigma Y, \sigma)-\frac{1}{Y} D_{i}(\sigma Y)+(2-\sigma) D_{i}^{\prime}(\sigma Y),
\end{aligned}
$$

where the final line uses the second equality in (17). Substituting for $D_{i}$, using $\phi_{i}=0$, and rearranging gives

$$
\left.\frac{\partial \phi_{i}}{\partial \sigma}\right|_{\phi_{i}=0}=Y A_{i 1}(\sigma Y, \sigma)-\frac{g_{i}^{\prime}(\sigma Y) \varphi_{i}(Y, \sigma)}{1-\sigma},
$$

where

$$
\begin{aligned}
\varphi_{i}(Y, \sigma) & =\left(2-2 \sigma+\sigma^{2}\right) u_{i}^{\prime}\left[R-g_{i}(\sigma Y)\right]-(1-\sigma)^{2} u_{i}^{\prime}\left[-g_{i}(\sigma Y)\right] \\
& =\sigma(2-\sigma) u_{i}^{\prime}\left[R-g_{i}(\sigma Y)\right]+(1-\sigma)^{2} d_{i}(\sigma Y) .
\end{aligned}
$$

Since $\sigma(2-\sigma)>0$ for $\sigma \in(0,1)$ and $d_{i} \geq 0$, we must have $\varphi_{i}>0$ if $\sigma>0$. Using (18), we can deduce that $\partial \phi_{i} / \partial \sigma<0$ when $\phi_{i}=0$, completing the proof.

Proof of Proposition 5.1. The equilibrium condition $1 / n \in \mathcal{S}\left(Y^{n}\right)$ is equivalent to $\phi\left(n y^{n}, 1 / n\right)=0$, where $y^{n}=Y^{n} / n$ and

$$
\phi\left(\frac{y}{\sigma}, \sigma\right)=A(y, \sigma)+\frac{(1-\sigma) \sigma}{y} D(y),
$$

from the definition in (9). (We drop subscripts throughout this proof.) Hence,

$$
\begin{aligned}
\sigma \frac{\partial}{\partial \sigma} \phi\left(\frac{y}{\sigma}, \sigma\right) & =\sigma \frac{\partial}{\partial \sigma} A(y, \sigma)+\frac{\sigma(1-2 \sigma)}{y} D(y) \\
& =A(y, \sigma)+g^{\prime}(y) u_{i}^{\prime}\left[-g_{i}(y)\right]+\frac{\sigma(1-2 \sigma)}{y} D(y)
\end{aligned}
$$

If $\phi(y / \sigma, \sigma)=0$, we find, with some manipulation,

$$
\begin{aligned}
& (1-\sigma) \sigma \frac{\partial}{\partial \sigma} \phi\left(\frac{y}{\sigma}, \sigma\right) \\
= & -\sigma^{2} g^{\prime}(y)\left\{u^{\prime}[R-g(y)]-u^{\prime}[-g(y)]\right\}+(1-2 \sigma) g^{\prime}(y) u^{\prime}[-g(y)] .
\end{aligned}
$$


Concavity of the utility function implies that the term in braces is nonpositive. So, considered as a function of $\sigma, \phi(y / \sigma, \sigma)$ crosses the axis in the interval $[0,1 / 2]$ at most once and from below.

Since $\phi\left(n y^{n}, 1 / n\right)=0$, we have $\phi\left((n+1) y^{n}, 1 /(n+1)\right)<0$. Also,

$$
\phi\left((n+1) y^{n+1}, \frac{1}{n+1}\right)=0,
$$

so Lemma 9.1 implies that $y^{n}>y^{n+1}$.

Proof of Lemma 5.1. Under the assumptions of the lemma, contestant $i$ has a share function $s_{i}$, which satisfies the properties set out in Lemma 4.1. Indeed, if $s_{i}(Y)=0$, then $s_{i}(\widetilde{Y})=0$ and the conclusion of the lemma follows trivially.

For the case when $s_{i}(Y)>0$, we first observe that $s_{i}$ is strictly decreasing where positive, so

$$
Y\left[1-s_{i}(Y)\right]<\tilde{Y}\left[1-s_{i}(\tilde{Y})\right] .
$$

The definition of a share functions says that $Y s_{i}(Y)$ is a best response to $Y_{-i}=Y-Y s_{i}(Y)$. Hence,

$$
\begin{aligned}
\pi_{i}\left(Y s_{i}(Y), Y\right) & =\max _{y \geq 0} \pi_{i}\left(y, Y-Y s_{i}(Y)+y\right) \\
& >\max _{y \geq 0} \pi_{i}\left(y, \widetilde{Y}-\widetilde{Y} s_{i}(\widetilde{Y})+y\right) \\
& =\pi_{i}\left(\widetilde{Y} s_{i}(\widetilde{Y}), \widetilde{Y}\right) .
\end{aligned}
$$

The inequality in the second line follows from the fact that $\pi_{i}(y, Y)$ is a strictly decreasing function of $Y$ for any $y>0$.

Proof of Lemma 6.1. Since $u_{i}^{\prime}$ is convex, the area under its graph is smaller than the area under the chord from $-g(y)$ to $R-g(y)$, giving the inequality [9]:

$$
\frac{1}{2} u_{i}^{\prime}[R-g(y)]+\frac{1}{2} u_{i}^{\prime}[-g(y)] \geq \frac{u_{i}[R-g(y)]-u_{i}[-g(y)]}{R},
$$

for any $y \geq 0$. Since $u_{i}^{\prime}$ is decreasing, we deduce that, if $\sigma \leq 1 / 2$, then $A_{i}(y, \sigma) \leq-g_{i}^{\prime}(y) D_{i}(y) / R$. It follows from (9) that

$$
\phi_{i}(Y, \sigma) \leq \frac{D_{i}(\sigma Y)}{R} \phi_{i}^{\mathrm{N}}(Y, \sigma) .
$$


Since $\sigma \in \mathcal{S}_{i}(Y)$ implies $\phi_{i}(Y, \sigma)=0$ if $\sigma<1$ (the case $\sigma=0$ is trivial), we conclude that $\phi_{i}^{\mathrm{N}}(Y, \sigma) \geq 0$. Since $\phi_{i}^{\mathrm{N}}\left(Y, s_{i}^{\mathrm{N}}(Y)\right)=0$ and $\phi_{i}^{\mathrm{N}}$ is strictly decreasing (since risk neutrality implies regularity), we have $s_{i}^{\mathrm{N}}(Y) \geq \sigma$.

If contestant $i$ is also strictly risk averse, then $u_{i}^{\prime}[R-g(y)]>u_{i}^{\prime}[-g(y)]$ for all $y$. If $\sigma<1 / 2,(21)$ holds strictly and $\phi_{i}(Y, \sigma)=0$, then $\phi_{i}^{\mathrm{N}}(Y, \sigma)>0$ and the final assertion of the lemma follows directly.

The proof of Lemma 6.2 exploits the following lemma, which can also be used to establish Proposition 6.2 when contestants are not regular.

Lemma 9.2 Suppose the production function $f$ satisfies $\boldsymbol{A} 1$ and $f^{\prime}(0)<\infty$ and the utility function $u$ satisfies $\boldsymbol{A} 2$. Let $\mathcal{S}$ be the corresponding share correspondence. For any $Y^{0} \in(0, \bar{Y})$, there is a $\nu\left(Y^{0}\right)>0$, such that $\sigma \geq \nu\left(Y^{0}\right)$ for any $\sigma \in \mathcal{S}(Y)$ and any $Y \in\left(0, Y^{0}\right]$.

When regularity holds, there will be a share function $s$ and the lemma is trivial: by Lemma 4.1, the share function is positive and strictly decreasing in $(0, \bar{Y})$ and we only have to take $\nu\left(Y^{0}\right)=s\left(Y^{0}\right)$. The proof is slightly more intricate with a share correspondence, since this is not necessarily decreasing.

Proof of Lemma 9.2. Part 3 of Lemma 3.2 implies that there is a $Y^{*} \in(0, \bar{Y})$ such that $\sigma>1 / 2$ for all $Y \in\left(0, Y^{*}\right)$. Now define

$$
\begin{aligned}
\eta\left(Y^{0}\right) & =\inf \left\{\sigma: \sigma \in \mathcal{S}(Y), Y \in\left[Y^{*}, Y^{0}\right]\right\} \\
& =\inf \left\{\sigma: \phi_{i}(Y, \sigma)=0, Y \in\left[Y^{*}, Y^{0}\right]\right\} .
\end{aligned}
$$

Since $Y^{0}<\bar{Y}$, the equivalence of the two definitions is a direct consequence of Part 2 of Lemma 3.2 and the first-order conditions characterizing $\mathcal{S}$. Since $\phi_{i}$ is continuous in both arguments, the infimum is achieved at $(\widetilde{Y}, \widetilde{\sigma})$, say and $\widetilde{\sigma} \in \mathcal{S}(\widetilde{Y})$, so $\widetilde{\sigma}>0$, by Lemma 3.2. By construction, if $Y \in\left[Y^{*}, Y^{0}\right]$ and $\sigma \in \mathcal{S}(Y)$, then $\sigma \geq \widetilde{\sigma}$.

To complete the proof, note that, if $Y^{0} \leq Y^{*}$, the lemma holds with $\nu\left(Y^{0}\right)=1 / 2$. If $Y^{0}>Y^{*}$, the lemma holds with $\nu\left(Y^{0}\right)=\min \{1 / 2, \tilde{\sigma}\}$.

Proof of Lemma 6.2. For each $n$, we let $\widehat{Y}^{n}$ be an equilibrium value of $Y$ in $\mathcal{C}^{n}$ and first show that $\widehat{Y}^{n} \longrightarrow \bar{Y}$ as $n \longrightarrow \infty$. Part 2 of Lemma 3.2 implies that $\widehat{Y}^{n}<\bar{Y}$. If $Y^{0} \in(0, \bar{Y})$, it follows from Lemma 9.2 that, if $n>1 / \nu\left(Y^{0}\right)$ and $\sigma_{i} \in \mathcal{S}(Y)$ for all $i$, where $Y<Y^{0}$, then $\sum_{j=1}^{n} \sigma_{j}>1$. Therefore there cannot be an equilibrium value of $Y \in\left(0, Y^{0}\right]$. Hence $\widehat{Y}^{n}>Y^{0}$ for all large 
enough $n$, as claimed. For all $Y>0$, define $\bar{\eta}(Y)=\max \mathcal{S}(Y)$. Part 2 of Lemma 3.2, implies that $\bar{\eta}(Y) \longrightarrow 0$ as $Y \longrightarrow \bar{Y}$ and therefore

$$
\bar{\eta}\left(\widehat{Y}^{n}\right) \longrightarrow 0 \text { as } n \longrightarrow \infty \text {. }
$$

To complete the proof, note that the dissipation ratio corresponding to the equilibrium $\widehat{\mathbf{y}}^{n}$ is

$$
\rho^{n}=\frac{1}{R} \sum_{j=1}^{n} g\left(\widehat{\sigma}_{j}^{n} \widehat{Y}^{n}\right),
$$

where $\widehat{\sigma}_{i}^{n}=\widehat{y}_{i}^{n} / \widehat{Y}^{n}$ for all $i$. By the Intermediate Value Theorem, there is $\theta_{i}^{n} \in[0,1]$ for each $i$ such that

$$
g\left(\widehat{\sigma}_{i}^{n} \widehat{Y}^{n}\right)=g(0)+\widehat{\sigma}_{i}^{n} \widehat{Y}^{n} g^{\prime}\left(\theta_{i}^{n} \widehat{\sigma}_{i}^{n} \widehat{Y}^{n}\right)
$$

for all $n$ and $i$. Since $g(0)=0$ and $g^{\prime}$ is a non-decreasing function,

$$
\rho^{n} \geq \frac{1}{R} \sum_{j=1}^{n} \widehat{\sigma}_{j}^{n} \widehat{Y}^{n} g^{\prime}(0)=\frac{\widehat{Y}^{n}}{R} g^{\prime}(0),
$$

using the equilibrium condition $\sum_{j=1}^{n} \widehat{\sigma}_{j}^{n}=1$, and

$$
\begin{aligned}
\rho^{n} & =\frac{1}{R} \sum_{j=1}^{n} \widehat{\sigma}_{j}^{n} \widehat{Y}^{n} g^{\prime}\left(\theta_{j}^{n} \widehat{\sigma}_{i}^{n} \widehat{Y}^{n}\right) \\
& \leq \frac{1}{R} \sum_{j=1}^{n} \widehat{\sigma}_{j}^{n} \widehat{Y}^{n} g^{\prime}\left(\bar{\eta}\left(\widehat{Y}^{n}\right) \widehat{Y}^{n}\right) \\
& =\frac{\widehat{Y}^{n}}{R} g^{\prime}\left(\bar{\eta}\left(\widehat{Y}^{n}\right) \widehat{Y}^{n}\right),
\end{aligned}
$$

where the inequality uses $\theta_{j}^{n} \sigma_{j}^{n} \leq \sigma_{j}^{n} \leq \bar{\eta}\left(\widehat{Y}^{n}\right)$. We can deduce that $\rho^{n} \longrightarrow g^{\prime}(0) \bar{Y} / R=\widetilde{\rho}[u]$ as $n \longrightarrow \infty$ from $\widehat{Y}^{n} \longrightarrow \bar{Y}$ and $(22)$. 